\title{
Dynamics and chemistry of SL9 plumes
}

\author{
By KEVIN ZAHNLE
}

\author{
NASA Ames Research Center, M.S. 245-3, Moffett Field, California 94035-1000, USA
}

The SL9 impacts are known by their plumes. Several of these were imaged by HST towering $3000 \mathrm{~km}$ above Jupiter's limb. The heat released when they fell produced the famous infrared main events. The reentry shocks must have been significantly hotter than the observed color temperature would imply, which indicates that the shocks were radiatively cooled, and that most of the energy released on reentry was radiated. This allows us to use the infrared luminosities of the main event to estimate the energy of the impacts; we find that the $R$ impact released some $0.3-1 \times 10^{27}$ ergs. Shock chemistry generates a suite of molecules not usually seen on Jupiter. The chemistry reflects a wide range of different shock temperatures, pressures, and gas compositions. The primary product, apart from $\mathrm{H}_{2}$, is $\mathrm{CO}$, the yield of which depends only weakly on the comet's composition, and so can be used to weigh the comet. Abundant water and $\mathrm{S}_{2}$ are consistent with a somewhat oxidized gas (presumably the comet itself), but the absence of $\mathrm{SO}_{2}$ and $\mathrm{CO}_{2}$ shows that conditions were neither too oxidizing nor the shocks too hot. Meanwhile, production of $\mathrm{CS}, \mathrm{CS}_{2}$, and $\mathrm{HCN}$ appears to require a source in dry jovian air; i.e., the airbursts occurred above the jovian water table. Tidal disruption calculations and models of the infrared light curves agree on an average fragment diameter of about half a kilometer. Chemical products and atmospheric disruption models agree on placing the terminal explosions around the 1 to 4 bar levels. The plumes were spectacular because the explosions were shallow, not because the explosions were large.

\section{Introduction}

Nine months after P/Shoemaker-Levy 9 (SL9) struck Jupiter, the size of the fragments and the depth of their terminal airbursts remain controversial. In this chapter I will be concerned mostly with the rise and fall of the ejecta plumes. I will address six topics. The first is a brief overview of the observed light curves, with interpretation and foreshadowing as seems appropriate. This is followed by an illustrative summary of a detailed numerical simulation of a 2-D, axisymmetric ejecta plume. The numerical model shows several of the observed phenomena directly (had we heeded our model, our predictions would have been better), but as it lacks radiative cooling, it can't be used to calculate light curves. To explain the numerical simulations we developed a simple analytic approximation that can be used to calculate light curves (Zahnle \& Mac Low, 1995) - how this is done is the third topic. The fourth topic is shock chemistry. Chemical products sample the composition of the most strongly shocked air, and thereby constrain the composition of the comet and the depth of the explosions. Fifth, we address the nature of the $450 \mathrm{~m} \mathrm{~s}^{-1}$ wave, which, if interpreted as a deep tropospheric gravity wave, could provide an observational argument that might favor deep explosions (cf., Ingersoll \& Kanamori 1995). Finally, we offer an explanation of why the plumes were all the same height, and in the process make the point that big plumes do not require big impactors.

\section{Light curves}

As viewed at infrared wavelengths from Earth, a typical light curve had three peaks, the first two faint and brief and the third bright and long-lasting (Graham et al. 1995; Nicholson et al. 1995). Although first reported for the $\mathrm{R}$ impact (Graham et al. 1995), 


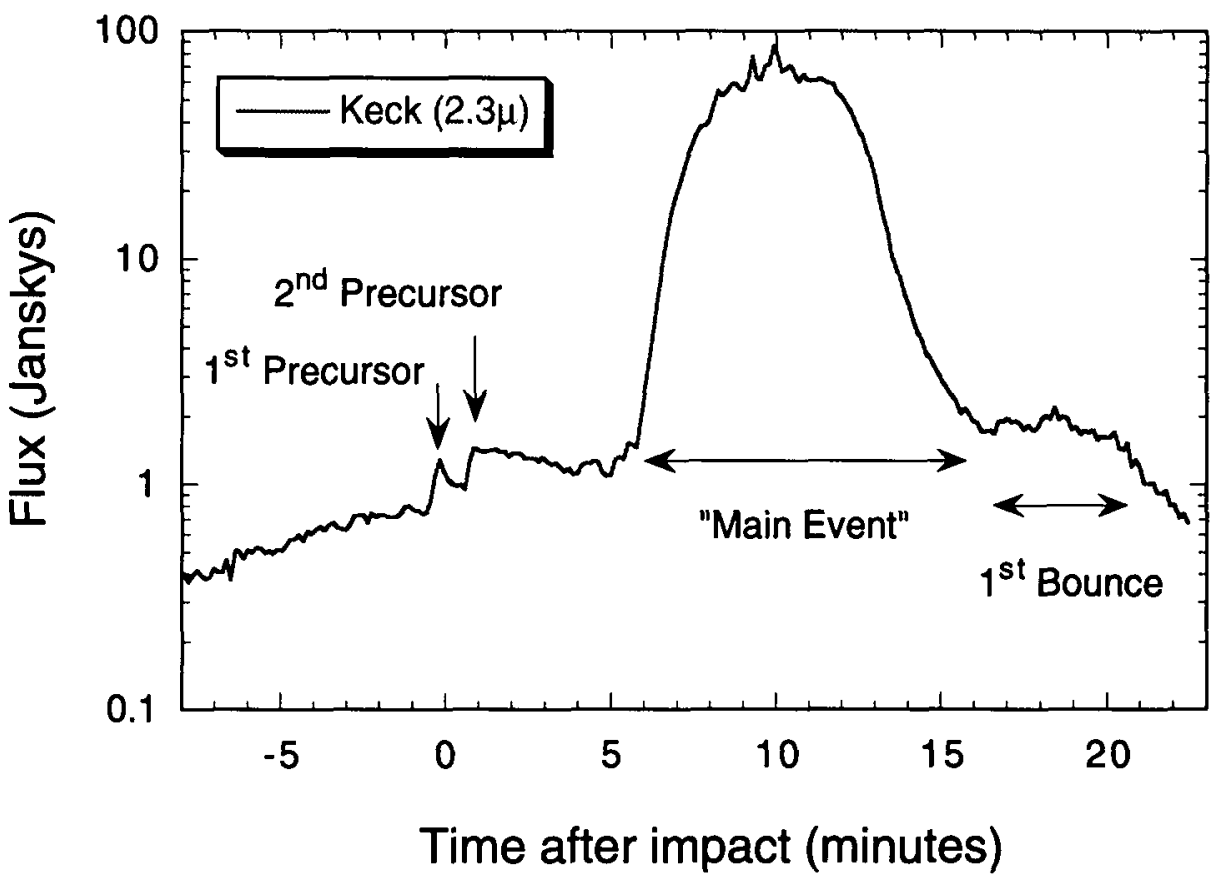

FIGURE 1. Annotated light curve of fragment $R$ at $2.3 \mu$, after Graham et al. (1995).

the three peaks were seen for all the well-observed events (Nicholson, this volume, provides what will probably remain the definitive summary of SL9 light curves). But because the first and second peaks were subtle, and so mostly overlooked at the time, they have since been called the first and second precursors. The spectacular third peak (or "main event") was seen by all, often in real time, thanks to the Internet. By contrast, the Galileo spacecraft, which was well placed to view the events directly, detected two peaks of comparable magnitude, the first corresponding roughly with the first precursor, and the second corresponding with the main event.

Figure 1 shows a typical SL9 light curve (shown is the Keck curve for fragment $R$ at 2.3 microns, Graham et al. 1995). Figure 2 is a cartoon that illustrates the viewing geometries and evolution of a typical event. The first peak, or first precursor, signaled the entry of the fragment into Jupiter's atmosphere. A typical first precursor was detected on Earth some 10 seconds before the impact was first detected by the Galileo spacecraft. This apparent paradox was resolved at this conference, and is discussed in detail in the chapter by Chapman. In brief, it appears that the meteor trail was first seen from Earth while it was still high enough above Jupiter's cloudtops to be viewed directly by terrestrial observers. This early signal was too faint to be detected by Galileo. Only later, well after the meteor disappeared behind the limb as viewed from Earth, did it become bright enough to be detected by Galileo. From either vantage the characteristic time scale of brightening was short, consistent with the rise time of order $H / v \sim 1 \mathrm{~s}$ expected for a meteor of velocity $v$ penetrating an atmosphere of scale height $H$. That the first precursor was seen at infrared wavelengths implies a relatively low temperature for the emitting matter; this is most easily understood as the exploding meteor trail, in which gas cools by expansion on a timescale of tens of seconds.

Most of the meteor's initial kinetic energy is released in the last second of its existence. These matters are discussed in detail in the chapters by Mac Low and Crawford. What 


\section{Galilio $P$}

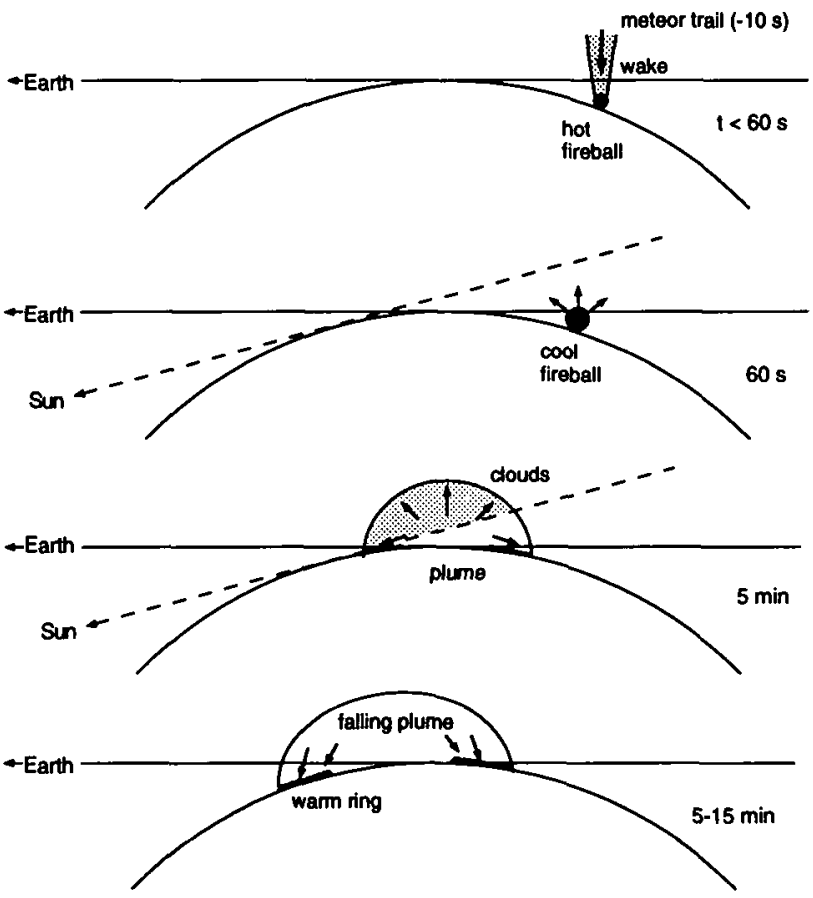

FIgURE 2. A cartoon that illustrates the viewing geometry of a typical SL9 impact. The first precursor corresponds to the fragment entering the atmosphere. The second precursor corresponds to the fireball rising into direct view from Earth. HST observed the plume in scattered sunlight, projected against the black sky of space. Finally, when the plume fell back to Jupiter, it produced a bright infrared event seen well from both Galileo and Earth.

is important here is that at the end of its flight the meteor releases enough energy in a small enough volume that the result is an explosion.

The second peak, or second precursor, occurs when the fireball from the terminal explosion rises above Jupiter's limb into direct view from Earth. That this would happen was predicted (Boslough et al. 1994; Ahrens et al. 1994). By the time ( $\sim 60 \mathrm{~s})$ the fireball cleared the limb it had cooled to $\sim 500-700 \mathrm{~K}$ (Carlson et al. 1995a). Accordingly, the second peak was more prominent at longer wavelengths, as is immediately apparent when the Palomar light curves (3.2 and $4.5 \mu \mathrm{m}$, Nicholson et al. 1995a) and the Keck light curve $(2.3 \mu \mathrm{m}$, Graham et al. 1995$)$ for the R impact are compared. In one of their numerical simulations, Boslough et al. (1994) get both the timing and the temperature of the debris front about right for a $1 \mathrm{~km}$ (diameter) impactor.

The onset of the second peak was abrupt (Graham et al. 1995), which requires the fireball to have a sharply defined photosphere when it rises above the limb. The observed rise time, $\sim 7 \mathrm{~s}$, is what one would expect from a $100-200 \mathrm{~km}$ diameter fireball rising at $13 \mathrm{~km} \mathrm{~s}^{-1}$. A well-defined surface is a general feature of numerical fireballs produced by terminal explosions (e.g., Zahnle \& Mac Low 1994; Boslough et al. 1994). The more evenly distributed line charge has an initial length scale of order $H$, which is mostly forgotten by the time the fireball inflates to $>100 \mathrm{~km}$ diameter. 
As the fireball rises it continues to expand, cool, and fade. In the fireball are found a fair fraction of the former comet and a comparable mass of highly shocked Jovian air. The fireball begins as the hottest part of the ejecta plume. It rises the fastest, and eventually becomes the vanguard of the plume. A much larger mass of mostly jovian air follows behind. Because the fireball's radiating surface is relatively small, radiative losses are small, and the ejecta plume expands almost adiabatically. Most of the plume's initial thermal energy is converted to kinetic energy of expansion. As the fireball expands first silicates condense, later carbonaceous matter, then water (if present), ammonia, and so on. The condensates made the sunlit parts of the plume visible from Earth, there to be imaged by the Hubble Space Telescope (HST) (Hammel et al. 1995).

The third peak was caused by the ejecta plume falling back on the atmosphere (Zahnle \& Mac Low 1995; Nicholson et al. 1995; Graham et al. 1995). The infrared events typically began about 5 or 6 minutes after the impact itself, and lasted another 10-15 minutes, somewhat less for the smallest events (e.g., D), and somewhat more for the largest (e.g., $\mathrm{K})$. The timescales are those expected for a falling ballistic plume. It takes about $\sqrt{8 z / g}$ for a plume to rise to a height $z$ and fall back again. For the $3000 \mathrm{~km}$ plumes observed by the HST, this time is $\sim 10^{3} \mathrm{~s}$, i.e., 15 minutes. In the Palomar and Keck data the main light peak was followed by two smaller maxima about 10 and 20 minutes later (Nicholson et al. 1995), probably bounces of some sort (see also chapter by Mac Low). Presumably these too were universal features.

Because the plume falls over an enormous area, thermal radiation produced by the plume's reentry into the atmosphere accounts for a large fraction of the impact energy. In our numerical simulations the plume's kinetic energy is typically about $20-40 \%$ of the total impact energy; the higher fraction is seen in the models with the best energy conservation. That most of this was promptly radiated is implicit in the low effective radiating temperature, roughly 500 to $1000 \mathrm{~K}$ (Nicholson, this volume). These temperatures are much lower than shock temperatures obtained using the standard Rankine-Hugoniot jump conditions. Hot shocks were real enough: appropriately high temperatures (2000$5000 \mathrm{~K}$ ) are seen in the 2.3 micron CO band (Knacke et. al. 1994, Crisp \& Meadows 1995, Kim et al. 1995). But most of the radiation is much cooler. Apparently radiative cooling of the shocked material was efficient. One plausible opacity source-dust-is clearly visible in the Hubble images. Another is line emission by very hot molecules. To some extent the latter surely occurred, as the aforementioned high $\mathrm{CO}$ temperatures indicate.

Although it is natural to think that the 5-6 minute delay between an impact and the main event was at least in part due to rotation of the impact site into better view, it now appears that viewing geometry was relatively unimportant. This is shown by the timing of the IR light curve recorded by Galileo for the $R$ and $G$ events, which is similar to that observed on Earth (Carlson et al. 1995b). This implies that something other than Jupiter's rotation delayed the third peak.

One possibility is that very little material was ejected with vertical velocities between 2 and $5 \mathrm{~km} \mathrm{~s}^{-1}$. Although not a hypothesis I am fond of, it might be consistent with a rising fireball: we do see evidence in our 2-D numerical simulations for a modest lack of ejecta with velocities between 2 and $7 \mathrm{~km} \mathrm{~s}^{-1}$ (see below), but not the near-total absence required to account for the relatively abrupt onset of the main events. It is imaginable that a more realistic geometry might alter this in the desired direction.

But our favorite hypothesis is that emission required dust, and dust did not appear in the ejecta blanket until 5-6 minutes had passed. One possibility is that the dust is cometary debris, either organic (cf., West et al. 1995) or silicate (cf., Field et al. 1995). Cometary debris would be among the most highly shocked material and therefore it would be among the fastest moving material in the ejecta plume. Another is that the dust is 
shock-generated organic material (cf., West et al. 1995). Shocks faster than about 4$5 \mathrm{~km} \mathrm{~s}^{-1}$ will generate carbonaceous dust from jovian air (Zahnle et al. 1995; the relevant shock chemistry will be discussed below). Shock chemistry can occur in the explosion or on reentry into the atmosphere, but in either case the affected gas spends some time aloft. As it takes about $2 v_{z} / g \approx 5$ minutes for $4 \mathrm{~km} \mathrm{~s}^{-1}$ ejecta to reenter the atmosphere, the shock-generation model predicts the timing of the third peak automatically.

A related possibility is that the radiating temperature was set directly by the condensation temperature of organic grains. The pressure and temperature of the reentry shock are roughly those of the graphite stability field in solar composition gas; i.e., where $\mathrm{CO}$ and $\mathrm{CH}_{4}$ are present in comparable abundance and the population of complex hydrocarbons peaks.

Any of these stories would be consistent with the morphology of the ejecta blanket: a dark outer crescent (or ring) encompassing a relatively dust-free interior. In a ballistic model the more distant material falls later, so that the inner edge of the outer crescent maps to the beginning of the main event.

\section{Numerical plumes}

Three dimensional numerical plume models have been presented by the Sandia group (Boslough et al. 1994; Crawford et al. 1994), Shoemaker et al. (1995), and by Takata et al. (1994). The former models use an Eulerian grid, the last uses smoothed particle hydrodynamics (SPH). Finite computational resources have restricted 3-D modelling to the first 2-3 minutes of an event (a 3-D plume model strains even the mammoth Paragon computer at Sandia). All three groups use initial conditions that place the explosions too deep, and as a consequence, the computed plumes are too small for a given impact. However, because these groups mostly modelled big impacts, many of the simulated plumes turned out to be about the right size; e.g., Takata et al. (1994) even predicted that the SL9 plumes would be $3000 \mathrm{~km}$ high (which they were). Plume height will be addressed in $\S 7$ below.

The 3-D models show a pronounced "wake effect": ejecta are launched preferentially along the bolide's entry path. This occurs because the wake is hot and rarefied, hence sound speeds (and shock speeds) are faster, and there is less inertia to slow expansion. That the ejecta would be preferentially channelled by the wake should be counted as a successful prediction. HST images clearly show that the ejecta blankets were azimuthally asymmetric, and thickest in the general direction from which the comets came (Hammel et al. 1995).

But the analogy to a cannon has been somewhat overdrawn. Even though mass was preferentially channelled through the wake, the velocity fields of the plumes were much more isotropic, and probably not greatly different from the velocity field of an axisymmetric plume. In particular, detailed examination of the HST images shows that the apex of the plume remained pretty much directly above the impact site throughout a plume's evolution (K. L. Jessup, pers. comm. 1995). This implies that the material shot straight up had the highest vertical velocity. By contrast, the apex of a cannonball plume moves downstream with time. Another argument that favors an isotropic velocity field is that the outer "crescent" appears to form a nearly complete, equidistant ring centered on the impact site, especially well seen in late observations of the fragment $\mathrm{K}$ event (McGregor et al. 1995).

Figures 3-6 show four stages in the evolution of a highly idealized 2-D axisymmetric numerical plume. The view is from the side. The gray scale indicates temperature, the contours indicate the fractional presence of cometary (fireball) material, and the arrows 


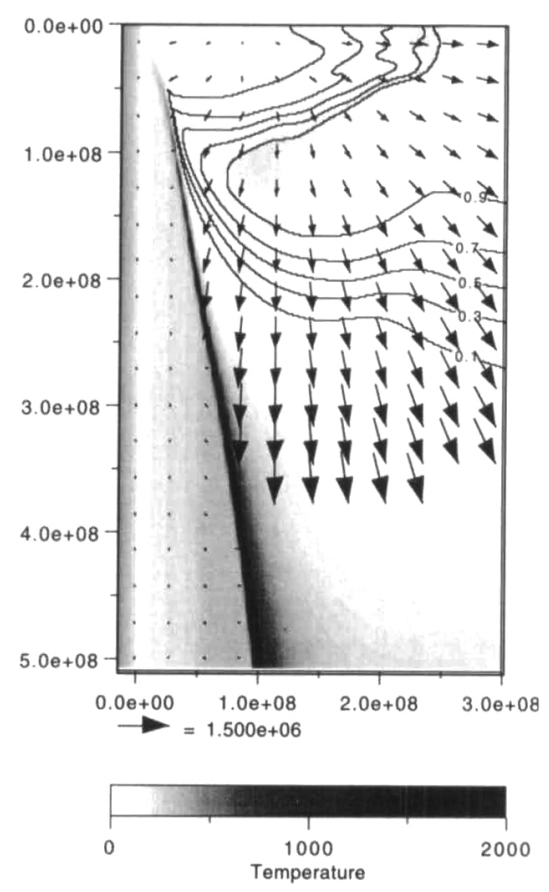

Figure 3. An axisymmetric numerical plume at 5.0, 6.7, 10.1, and 14.2 minutes (Fig. 3-6, respectively). The axes are labeled in $\mathrm{cm}$ and the velocities in $\mathrm{cm} \mathrm{s}$; the gray scale indicates approximate temperature in kelvins. The impactor's entry path is coincident with the left vertical axis; peak energy release (terminal explosion) is at $z=-25 \mathrm{~km}$ (2.4 bars). Contours mark the location of fireball material $(\sim 10 \%$ comet in this model). The shock wave from the explosion is prominent as a hot, wide cone, reaching $1000 \mathrm{~km}$ altitude $5000 \mathrm{~km}$ from the impact site. The former fireball is a discernable as a warm hemispheroid roughly $2000 \mathrm{~km}$ in radius. Underneath it is a cold plume of mostly jovian air.

indicate the velocity field. In this model the initial conditions presume a vertical line charge, coincident with the $z$-axis, extending from about $z=-50 \mathrm{~km}$ to the top of the grid (the altitude $z$ is measured from the 1 bar level), The resolution is $1 \mathrm{~km}$ near the site of the explosion, grading to $10 \mathrm{~km}$ in the most distant parts of the grid. The energy release profile is derived from an analytic approximation to large-body meteor flight (Zahnle \& Mac Low 1994). Peak energy release is at $-25 \mathrm{~km}$ ( $\sim 2.4$ bars) Most of the fragment's energy is released in the deepest scale height. The total energy release is $2 \times 10^{27} \mathrm{ergs}$, equivalent to a $750 \mathrm{~m}$ diameter fragment of density $0.5 \mathrm{~g} \mathrm{~cm}$. The size and density are consistent with the largest fragments produced by tidal disruption models of self-gravitating comets (Asphaug \& Benz 1994; Solem 1994; Asphaug \& Benz 1996). The detailed initial conditions and gridding for this model are described more fully in the chapter by Mac Low (this volume).

The contours mark the location of cometary material. However, the initial conditions were too coarse to retain this resolution, and as a result the comet is about ten-fold depleted in the fireball, so that a contour line of unity is only about $10 \%$ comet by mass. Although it seems reasonable to us that these highly shocked, turbulent gases would mix, we see little direct evidence for mixing in our numerical simulations (see Fig. 7 in chapter by Mac Low).

The hydrodynamic code computes mass density, energy density, and horizontal and vertical velocities: $\rho, e, v_{r}$, and $v_{z}$, respectively. These are the independent variables. Pressure $p$ is required to evaluate force; it is obtained from an equation of state. For simplicity we have assumed an ideal gas with constant $\gamma$ (ratio of specific heats), by 


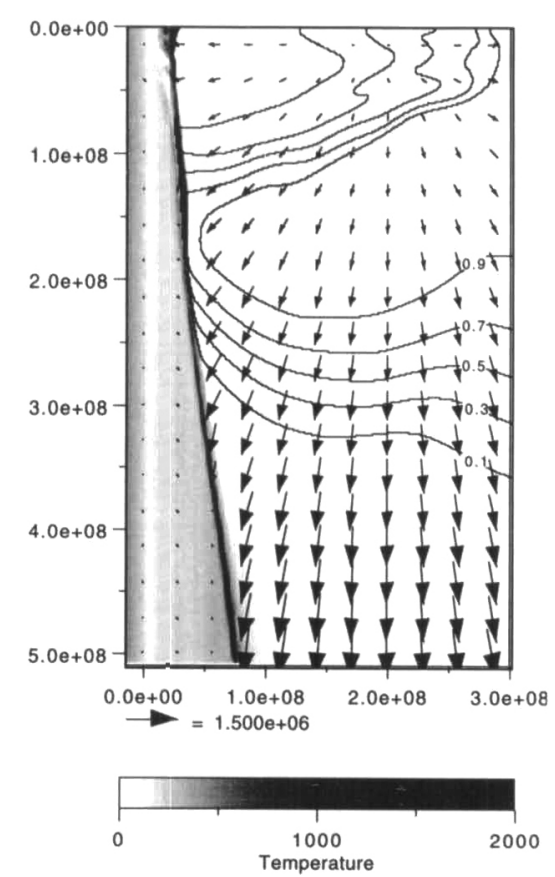

FIGURE 4. The axisymmetric numerical plume at 6.7 minutes. The reentry shock is prominent as an annulus $1000<r<2000 \mathrm{~km}$. It is warmest where the infalling material is richest in former comet.

which $p=e /(\gamma-1)$. In this simulation, which focuses on the dynamics of the plume, we take $\gamma=1.4$, which is appropriate to a cool diatomic gas. The dynamics do not appear to depend strongly on $\gamma$.

The hydrodynamics code computes neither temperature nor mean molecular weight; these require a more sophisticated equation of state, one that accounts for vaporization, dissociation, and ionization, for both jovian air and for cometary material. The latter presents myriad difficulties. The temperatures shown in Fig. 3-6 were computed assuming an ideal gas, $\left(\mu_{\mathrm{c}} \gg \mu_{\mathrm{J}}\right)$.

$$
T \approx \frac{p}{\rho} \frac{m_{\mathrm{H}}}{k}\left(\frac{1-f}{2.4}+\frac{f}{20}\right)^{-1}
$$

in which $m_{\mathrm{H}}$ is the mass of a proton; $\mu_{\mathrm{J}}=2.4$ and $\mu_{\mathrm{c}}=20$ are the mean molecular weights of jovian air and cometary gases respectively; and $f$ is the fraction that is cometary (by mass).

Temperatures computed using Eq. 3.1 are reasonably accurate for clean, cool jovian air, but become highly suspect for hot, comet-rich gas. There are three problems: (1) the mean molecular weight drops due to dissociation and ionization (e.g., $\mu_{\mathrm{J}}$ for dissociated air is about 1.3); (2) the composition of the comet-rich fireball is unknown, both because the composition of the comet is unknown, and because the degree of mixing with ambient air is unknown; and (3) radiative cooling becomes important. In Eq. 3.1, the mean molecular weight of cometary material is unimportant, because $f \leq 0.1$ sets an upper limit of $\mu=2.6$ on the mean molecular weight of the fireball. Shock temperatures reported by Kim et al. (1995) imply that the mean molecular weight was $\sim 2.5$. If mixing were much less efficient, then we would expect higher temperatures in the fireball material than shown on Figs. 3-6. 

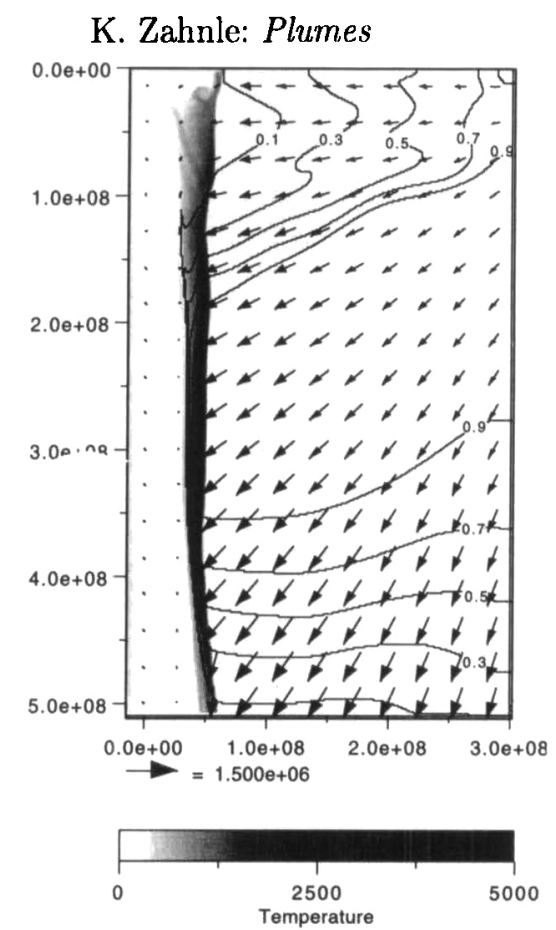

FIgURE 5. The axisymmetric numerical plume at 10.1 minutes. This is near peak light for the main event. The reentry shock is prominent as an annulus $2000<r<4000 \mathrm{~km}$; it is distinctly higher than the contact discontinuity between the plume material and the ambient jovian atmosphere. By this time the velocity field is almost precisely ballistic.

The most important omission from the numerical models is the exclusion of radiative cooling. As noted, radiative cooling was unimportant compared to adiabatic cooling during the explosion and during the expansion of the plume. The dynamics of the plume are reasonably well-described without it. But radiative cooling, either by molecules or by embedded or shock-generated particles, clearly dominated the energy budget of the reentry shock. We will return to radiative cooling in the next section, in the context of modelling the observed infrared light curves.

Figure 3 shows the plume 5 minutes after impact. The fireball material, found mostly in a thick hemispherical shell, by this time is no longer hot, but it has yet to fall back. Outside the shell are found hot but rarefied jovian gases that were shocked by the blast wave from the original explosion. Velocities in this gas are higher than predicted by homologous expansion; these high velocities recall the recent passage of the shock wave from the explosion. The shock itself is the prominent diagonal line of hot material (as a figure of revolution, it is really a wide cone). Inside the shell we find a fountain of extremely cold jovian air. This air had been heated by the explosion, but by this time has expanded adiabatically to a point where any condensibles within it have probably condensed.

Figure 4 , at $t=6.7$ minutes, shows the plume shortly after it has begun to fall back on the atmosphere. When the plume falls back onto the atmosphere, it liberates the vertical component of its kinetic energy in a reentry shock. There are actually two shocks, one driven into the Jovian atmosphere and the other into the falling plume. Warm shock temperatures are seen, especially between 1000 and $2000 \mathrm{~km}$ from the impact site. The highest temperatures occur in cometary gases, which have higher molecular weight. Warm temperatures are also seen at the epicenter, where jovian air reentering 


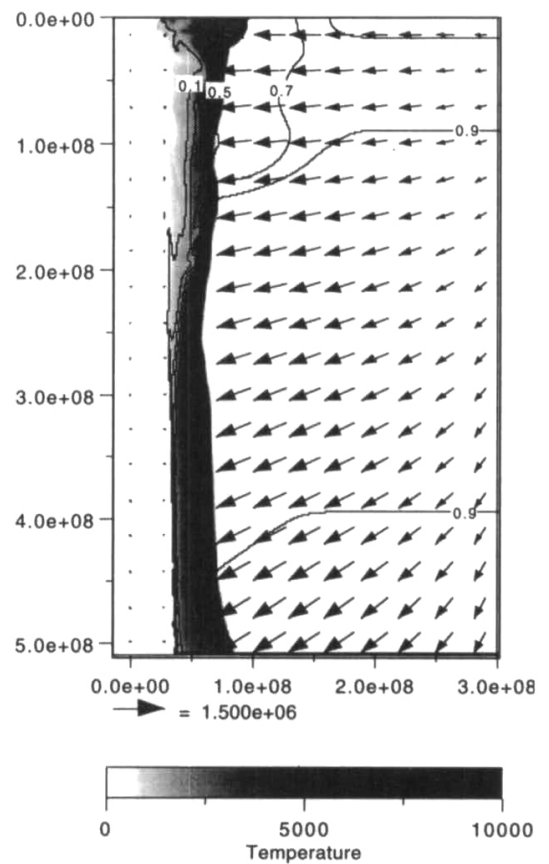

Figure 6. The axisymmetric numerical plume at 14.2 minutes. By this time the plume is fading. Because reentry velocities rise monotonically with time, the reentry shock is hotter than it has been, but so little material is falling that the shock is very high in the atmosphere, and no longer especially luminous. The contact discontinuity, marked by the lower contours, indicates the initial height of the reentry shock. The annulus as a whole is spreading rapidly. Not directly apparent is that it is also spreading homologously.

the atmosphere has been compressed and heated. By this time the velocity field in the plume is practically homologous and ballistic.

$$
v_{r}=\frac{r}{t} ; \quad v_{z}=\frac{z}{t}-\frac{1}{2} g t
$$

Note that the reentry shock has risen well above the the contact discontinuity (marked by the contours) between the shocked ejecta plume and the ambient jovian atmosphere.

By $t=10.1$ minutes (Fig. 5), the reentry shock has produced high temperatures in an annulus $1500<r<4000 \mathrm{~km}$ from the epicenter. Comet-rich material rains down on this annulus. Because reentry velocities get progressively higher as time passes, $v_{z} \approx \frac{1}{2} g t$, temperatures are higher.

Figure 6, at $t=14.2$ minutes, shows further evolution. Particularly clear in Fig. 6 is that (1) the shock temperatures are very high but that (2) the reentry shock has risen some $500 \mathrm{~km}$ above its original altitude, which is recalled by the position of the contact discontinuity between falling matter and ambient air. Relatively little material is actually falling by 14.2 minutes, which is why the shock is so high; indeed, previously shocked air is springing back at considerable velocity. These "bouncing shocks" may be responsible for the bouncing light curves seen so well in the Palomar data for the $\mathrm{R}$ impact (Nicholson et al. 1995; Nicholson, this volume; Deming et al. 1995; Mac Low, this volume).

Horizontal velocity is preserved across the shock. Therefore the shocked plume continues to expand radially for a considerable time after it reenters the atmosphere. This can be seen in Fig. 6; it is explicit in Fig. 7, which shows the position and velocity history of a "typical" parcel. One might expect the plume to expand horizontally until it has 


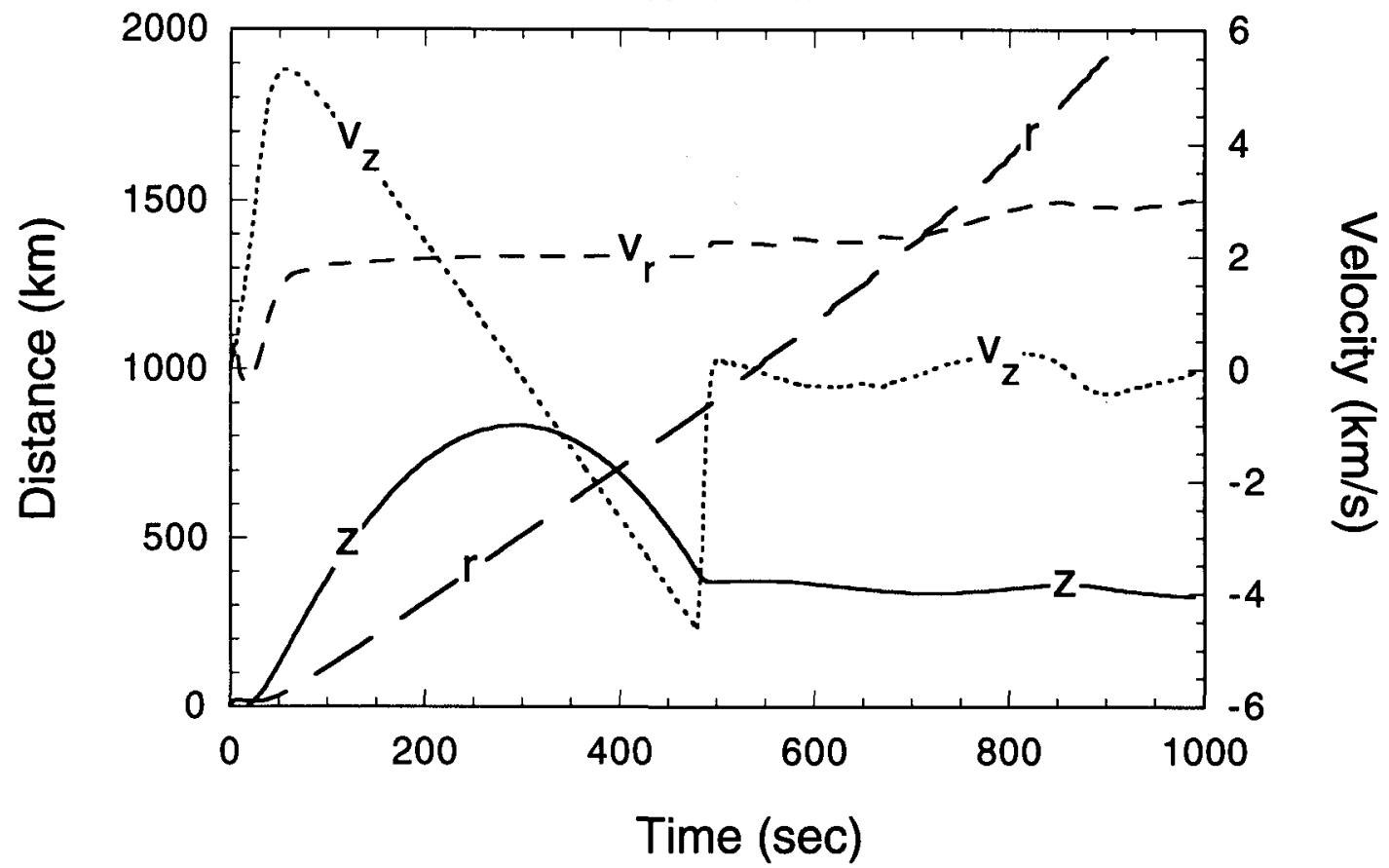

FIGURE 7. Location and velocity history of a representive strongly shocked parcel of jovian air. The parcel originated at the 2 bar level, near the site of the airburst. It was shocked to $T>9000 \mathrm{~K}$, expanded, cooled, and ultimately ejected at about $6 \mathrm{~km} / \mathrm{sec}$; it reentered the atmosphere $\sim 500 \mathrm{~s}$ later, where it was shocked. Note that the parcel retains its horizontal velocity across the shock.

swept up its own mass in jovian air. The characteristic crescent-shaped footprint of the plume was observed to have rotated through a larger angle than can be accounted for by Coriolis force acting only while the plume was in orbit (Hammel et al. 1995). The additional rotation of the footprint was caused by Coriolis force continuing to act on the radially expanding plume for another 20-30 minutes after reentry. An alternative perspective on the same process is that Jupiter rotated beneath the spreading plume until the plume coupled with the planet.

\section{A plume model}

As a first model for the infrared light curve, we (Zahnle \& Mac Low 1995) devised a 2-D axisymmetric plume model to describe the decline and fall of the ejecta plume. The "toy plume" model assumes that the velocity field of the ejecta plume is ballistic and that the mass-velocity distribution in the ejecta plume follows a power law that we have calibrated to our detailed numerical models. The ballistic approximation is valid to a factor of order $c_{s}^{2} / v^{2} \ll 1$, where $c_{s}$ is the sound speed and $v$ a typical velocity in the ejecta plume, and is in excellent agreement with the results of detailed numerical models. For simplicity we also assumed that the plume is axisymmetric with an opening angle $\theta^{\prime}$.

The mass-velocity distribution of ejecta from hypervelocity impacts generally obeys a power law of form

$$
M(>v) \propto v^{-\alpha}
$$

in which the notation $M(>v)$ refers to the cumulative mass ejected at velocities greater than $v$. Our numerical experiments of impacts on Jupiter give $\alpha \approx 1.6$ as an average over the range $0.01-10 \mathrm{~km} \mathrm{~s}^{-1}$, varying somewhat depending on explosion parameters. The 
distribution truncates at a maximum velocity, $v_{\max }$, that can be regarded as determining the height of the plume; typical SL9 plumes reached $3000 \mathrm{~km}$, so that $v_{\max } \approx 12 \mathrm{~km} \mathrm{~s}^{-1}$. The normalized form of Eq. 4.3 is

$$
M(>v) \approx 340 \eta m_{i}\left(\frac{1 \mathrm{~km} \mathrm{~s}^{-1}}{v}\right)^{1.55},
$$

where $\eta$ is the fraction of impact energy invested in the ejecta plume. Numerical simulations indicate that $\eta \approx 0.4$. Equation 4.4 has been evaluated for $\alpha=1.55$ and $v_{\max }=12 \mathrm{~km} \mathrm{~s}^{-1}$. These parameters describe the mass-velocity distribution we used in Zahnle \& Mac Low (1995), and in the subsequent discussion which is based on the paper.

However, application of a single power law to both strong $\left(v>c_{s}\right)$ and weak $\left(v<c_{s}\right)$ shocks is suspect, and when we examine our numerical models in detail we find that the plume is better described by two powers, for which $\alpha \approx 1.2$ for $v>c_{s}$ and $\alpha \approx 1.8$ for $v<c_{s}$. This distribution is not too far removed from momentum scaling for high velocities, and energy scaling for low velocities. As strong shocks and high velocity ejecta are our concern here, the lower value of $\alpha$ would have been a better choice. Such a distribution is relatively deficient in material launched with velocities $c_{s}<v<v_{\max } / 2$. which may have contrtibuted somewhat to the observed shapes of the main event light curves.

We subdivide the plume three dimensionally $(r, \theta, \phi)$ into a vast number of mass elements. Each element is launched on its own unique ballistic trajectory. We tile Jupiter's "surface" by distance and azimuth to produce a kind of dartboard centered on the impact site. We then count up where and when the mass elements reenter the atmosphere (i.e., where and when they hit the dartboard). The effective radiating temperature of the reentry shock is determined by balancing the energy supplied by infalling ejecta against thermal radiation by opacity sources (dust, soot, molecules, darts, etc.) embedded in or generated by the reentry shock. The approach is closely analogous to that used by Melosh et al. (1990) and Zahnle (1990) to model thermal radiation following the $\mathrm{K} / \mathrm{T}$ impact.

\subsection{Pressure}

The pressure level of the reentry shock, if strong, is

$$
p^{\prime}=\frac{\gamma+1}{2} \rho v_{z}^{2}
$$

This can be evaluated directly to give $p^{\prime}$ as a function of position and time, but this is not as useful or as accurate a measure of "the" shock pressure as one might hope. To first approximation, the greatest mass flux of material to arrive at any point is the first material to get there. Thereafter the mass flux declines monotonically and the shock rises to progressively lower pressures. Meanwhile the heated atmosphere both cools radiatively and also rises to seek a new scale height. The accumulated mass fallen at a given place (multiplied by $g$ ) provides a measure of the pressure level of the contact discontinuity between the plume and the Jovian atmosphere, which suggests that this is probably a good measure of the reentry shock.

An illustrative analytic approximation to the pressure at the contact discontinuity can be obtained by replacing $v^{2}$ in Eq. 4.4 by $r g$, which gives

$$
p(r)=\frac{g}{2 \pi r} \frac{d M}{d r}
$$




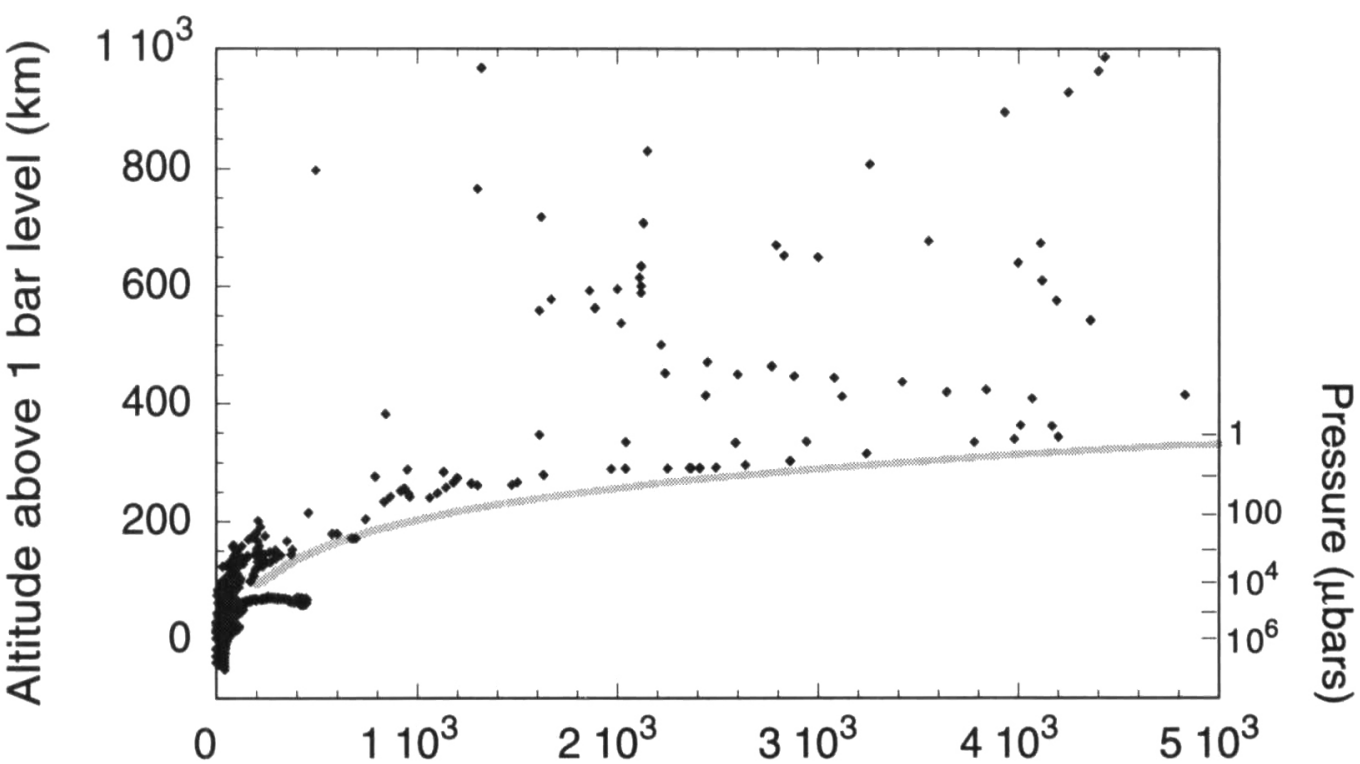

\section{Radial Distance from Impact Site $(\mathrm{km})$}

FIGURE 8. The altitude and pressure of the reentry shock $15 \mathrm{~min}$ after impact, for a $2 \times 10^{27} \mathrm{erg}$ SL9 impact (the model shown in Figs. 3-6). The solid curve shows the location of the reentry shock as approximated by Eq. 4.7. The diamonds show tracer particles that were swept up by the plume. The deepest diamonds trace the boundary between Jupiter and the fallen plume.

When evaluated for $\alpha=1.55$ and $v_{\max }=12 \mathrm{~km} \mathrm{~s}^{-1}$, this becomes

$$
p(r) \approx 90 \eta\left(\frac{m_{i}}{10^{14} \mathrm{~g}}\right)\left(\frac{1000 \mathrm{~km}}{r}\right)^{2.8} \mu \text { bars. }
$$

Shock pressures are directly proportional to the mass of the impactor. Pressures at large distances are quite low. At $r=2000 \mathrm{~km}$, a typical shock pressure produced by a $10^{14} \mathrm{~g}$ impact with $\eta=0.4$ would be $5 \mu$ bars.

Figure 8 compares the pressure level given by Eq. 4.7 (faded solid curve) to results obtained directly from the numerical model shown in Figs. 3-6 (diamonds). As this is the same numerical model by which we calibrated Eq. 4.4, good agreement might be expected. The diamonds indicate where tracer particles swept up in the plume have gone to after $15 \mathrm{~min}$. The lowermost diamonds trace the location of the boundary between Jupiter and the ejecta.

\subsection{Temperature}

The ordinary Rankine-Hugoniot relations for a strong shock require that the infalling material reach a temperature of

$$
T^{\prime} \geq \frac{\gamma-1}{2} \frac{m_{\mathrm{H}}}{k} v_{z}^{2}=1500 \mathrm{~K}\left(\frac{\mu}{2.5}\right)\left(\frac{v_{z}}{5 \mathrm{~km} / \mathrm{s}}\right)^{2},
$$

where $m$ is the mean molecular mass. The temperature in Eq. 4.8 is the temperature of the gas immediately after it passes through the shock. It can be high. Temperatures would be especially high in material that originated from the comet because the mean molecular weight of the vaporized comet is relatively large, probably of the order of $10<$ $\mu<20$. The detection of hot $(>2000 \mathrm{~K}$ ) carbon monoxide emission at $2.3 \mu \mathrm{m}$ (Knacke 
et al. 1994; Meadows et al. 1994; Crisp \& Meadows 1995, Kim et al. 1995) offers direct evidence that former cometary material became very hot on reentering the atmosphere. Figure 9 is a schematic picture of the shocks while the plume is still falling.

The effective radiating temperature, between 500 and $1000 \mathrm{~K}$ (Nicholson et al. 1995b), was much lower than implied by Eq. 4.8. Evidently radiative cooling by the shocked material was efficient. HST images clearly revealed that the ejecta blankets were dusty (Hammel et al. 1995). The dust is an obvious opacity source for radiative cooling. Another plausible source is cooling by molecular lines.

The dust could be cometary (ice, organic, or silicate), or it could be generated when the plume strikes the atmosphere. The latter requires a sufficiently strong shock. (Zahnle et al. 1995) show that reentry velocities must exceed $4-5 \mathrm{~km} \mathrm{~s}^{-1}$ for shock heating to be strong enough to burn jovian air. This could explain the relative transparency of the inner parts of the ejecta blanket as observed with the HST (Hammel et al. 1995), and the late onset of infrared radiation as observed by the Galileo spacecraft (Carlson et al. 1995a,b).

A simple expression for the radiating temperature of a dusty layer heated by falling ejecta is to assume an instantaneous energy balance between the kinetic energy of falling matter $(\dot{e})$ and radiative cooling, buffered by the heat capacity of the falling matter (Zahnle 1990):

$$
\dot{e}=\frac{1}{\gamma-1} \frac{k T}{m} \dot{m}+2 \sigma T^{4}\left(1-e^{-2 \tau}\right) .
$$

The factor $2 \sigma T^{4}$ assumes a grey radiator that emits both up and down (Fig. 9). The factor $1-e^{-2 \tau}$ allows for the optical depth $\tau$ of the radiating layer; the factor of 2 gives the right limit as $\tau \rightarrow 0$. We solve Eq. 4.9 for the temperature $T$ by Newton's method.

We consider two models for the optical depth $\tau$. Both assume a gray absorber that is uniformly embedded in the shocked air. In one model, $\tau$ is proportional to the total integrated mass of material falling at radius $r$, so that all the dust in the column contributes to radiative cooling. In the second model we limit $\tau$ to the most recently arriving material (specifically, only the most recent $90 \mathrm{~s}$ contribute). We do this because later arriving material shocks at progressively higher altitudes, for which the cumulative optical depth of deeper material may not be relevant. The temperatures that result are much more nearly constant, both in radius and time (Fig. 10).

The flux at Earth is calculated using $\tau(r, t)$ and $T(r, t)$, accounting for the rotation of Jupiter and the projected surface area of the plume's footprint. Figure 11 shows calculated light curves at 2.3, 3.2, and 4.5 microns, as seen from Earth. The Keck light curve for fragment R (Graham et al. 1995) at 2.3 microns is shown for comparison. The match at 3.2 microns and 4.5 microns is comparably good (Nicholson et. al. 1995). The important free parameters in the model are impact energy and opacity. The radiating temperature depends most strongly on opacity; impact energy scales the absolute flux.

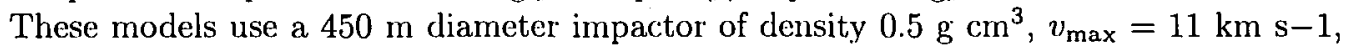
and opening angle $\theta^{\prime}=45^{\circ}$. The total impact energy is $5 \times 10^{26} \mathrm{ergs}, 40 \%$ of which is invested in the ejecta plume. The G, K, and L impacts were some 5-10 times brighter (Nicholson, this volume).

\section{Chemistry}

The impacts produced strong shocks, both promptly at the impact site and again, later, and over thousands of kilometers, when the ejecta plume reentered the atmosphere. The chemistry in these shocks was distinctive. The most surprising report was of a huge 


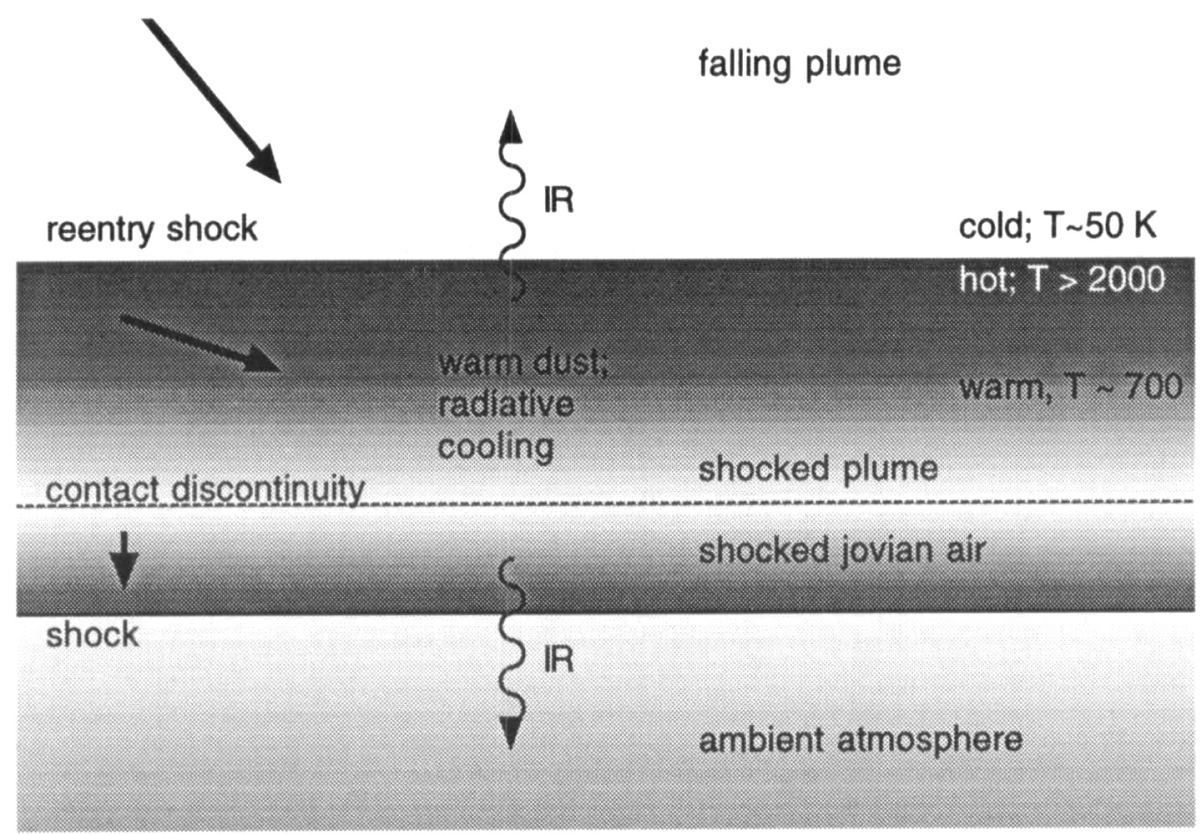

FIGURE 9. Radiative cooling in the reentry shocks. Velocities are shown schematically. There are two shocks, one driven into the ambient atmosphere and the other in the falling plume. The highest temperatures are in freshly shocked gas. Thereafter the gas cools radiatively. The contact discontinuity between air and plume is also a velocity discontinuity, as the ejecta continues to flow away from the impact site. Shear instabilities will cause mixing between atmosphere and ejecta, and eventually help brake the flow.

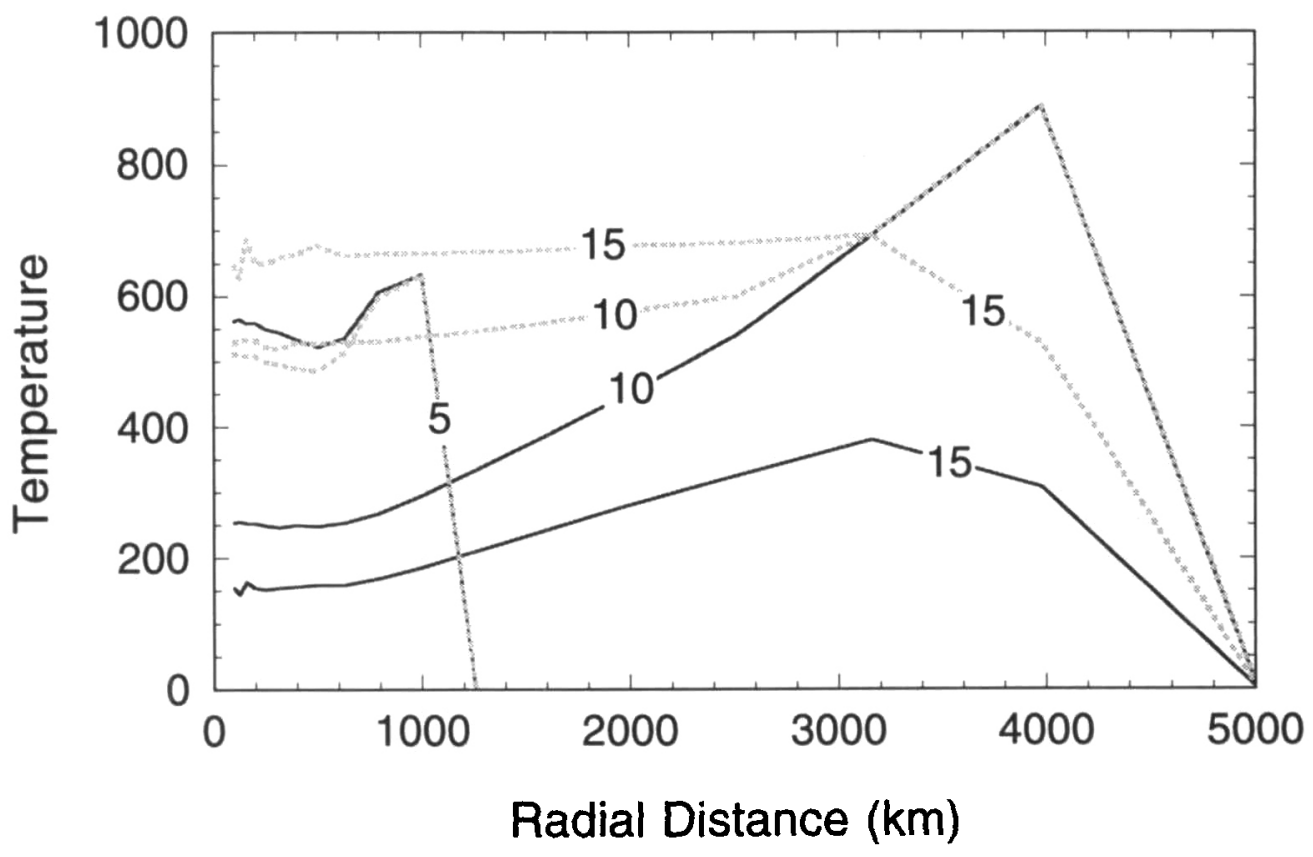

FIGURE 10. Effective radiating :emperatures at 5,10 , and 15 min produced by the toy plume with constant opacity $\kappa=100 \mathrm{~cm}^{2} \mathrm{~g}-1$. The solid curves use the total column optical depth. The dotted curves assume that only recently fallen material contributes to the effective optical depth. 


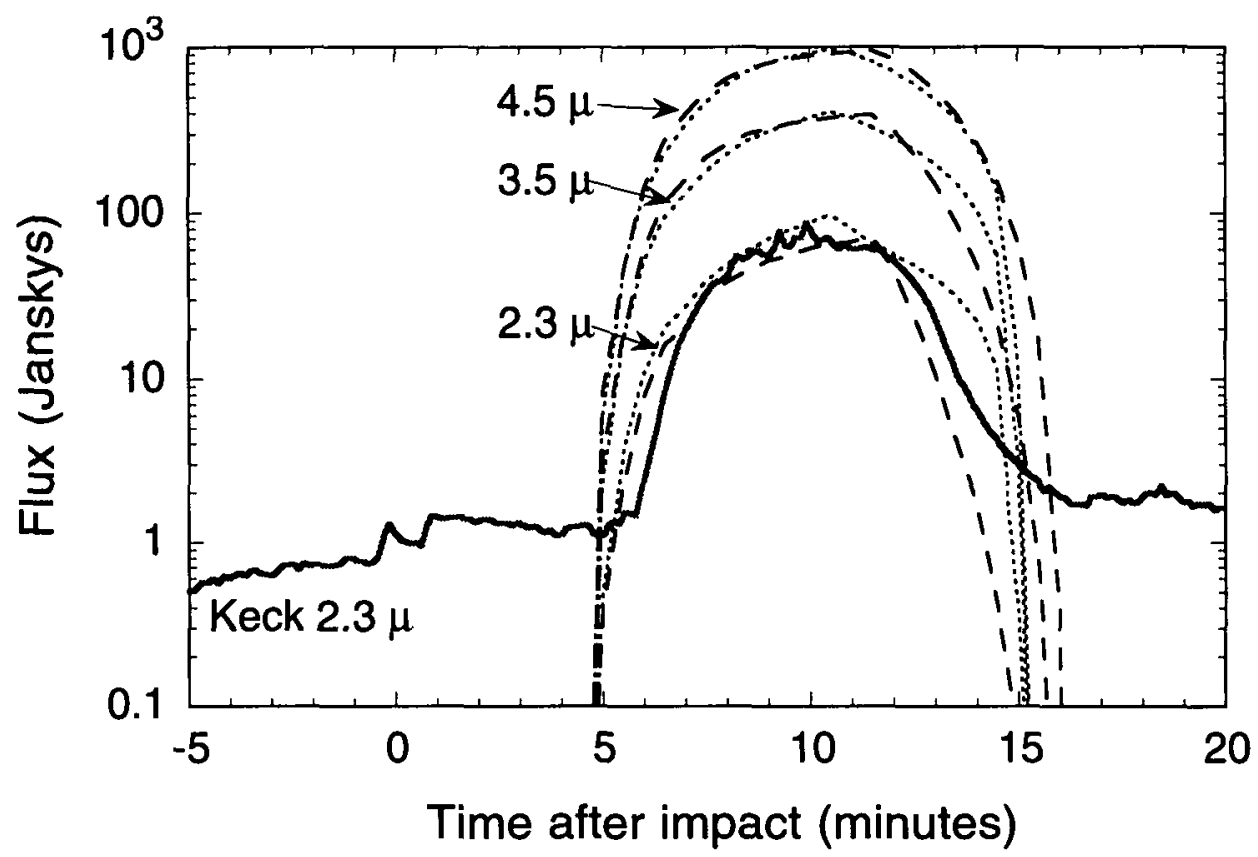

FIGURE 11. Light curves at selected infrared wavelengths generated by the toy plume model, as seen from Earth (Zahnle \& Mac Low 1995). The dashed curves assume that the total optical depth is the effective optical depth; the dotted curves assume that only recently fallen material contributes to the effective optical depth. The Keck light curve (Graham et al. 1995) at $2.3 \mu \mathrm{m}$ is shown for comparison (solid curve).

amount of diatomic sulfur $\left(\mathrm{S}_{2}\right)$ at the site of the $G$ impact (Noll et al. 1995). Other reported products unusual to Jupiter include CS, CS $2, \mathrm{OCS}, \mathrm{H}_{2} \mathrm{~S}, \mathrm{SO}_{2}, \mathrm{HCN}, \mathrm{CO}$, and $\mathrm{H}_{2} \mathrm{O}$ (Lellouch et al. 1995; Lellouch, this volume); aithough $\mathrm{H}_{2} \mathrm{~S}$ and $\mathrm{H}_{2} \mathrm{O}$ are doubtless abundant below the visible clouds.

A general rule of shock chemistry is that $\mathrm{CO}$ forms until either $\mathrm{C}$ or $\mathrm{O}$ is exhausted. If $\mathrm{O}>\mathrm{C}$, the other products are oxidized, and excess $\mathrm{O}$ goes to $\mathrm{H}_{2} \mathrm{O}$. If $\mathrm{C}>\mathrm{O}$, the other products are reduced, and excess $\mathrm{C}$ goes to $\mathrm{HCN} . \mathrm{C}_{2} \mathrm{H}_{2}$, and a wide variety of more complicated organics. Ultimately, given time, the carbon would react all the way to graphite, but in practice the reactions are incomplete. The dark ejecta debris is probably composed in part of carbonaceous particles generated by the shocks. In a sense, the SL9 impacts performed the farnous Miller-Urey experiment on a grand scale, with one result being the production of a lot of complex brown organic solids (called "tholins").

We use a straightforward chemical kinetics modei for the $\mathrm{H}, \mathrm{N}, \mathrm{C}, \mathrm{O}, \mathrm{S}$ system (Zahnle et al. 1995) to follow the nonequilibrium chemistry iehind the shocks. The model traces the evolving chemical composition of a parcel of gas by directly integrating the web of chemical reactions. Pressure and temperature histories of the parcels are patterned after those calculated by numerical hydrodynamic simuiations of the ejecta plume. A given plume parcel is generally shocked twice; i.e., a parcel shocked near the impact site is ejected at high velocity and is shocked again when is reenters the atmosphere. The final state of the gas depends mostly on the second shock. provided that the latter is hot enough. Figures 7 and 12 show an ilhustrative parcel history taken directly from the numerical model illustrated in Fig. 3-6.

We also used a thermochemical equilibrium mociel (Fegley \& Lodders 1994; Zahnle et al. 1995). The key difference between a kinctics model and a thermochemical cquilib- 


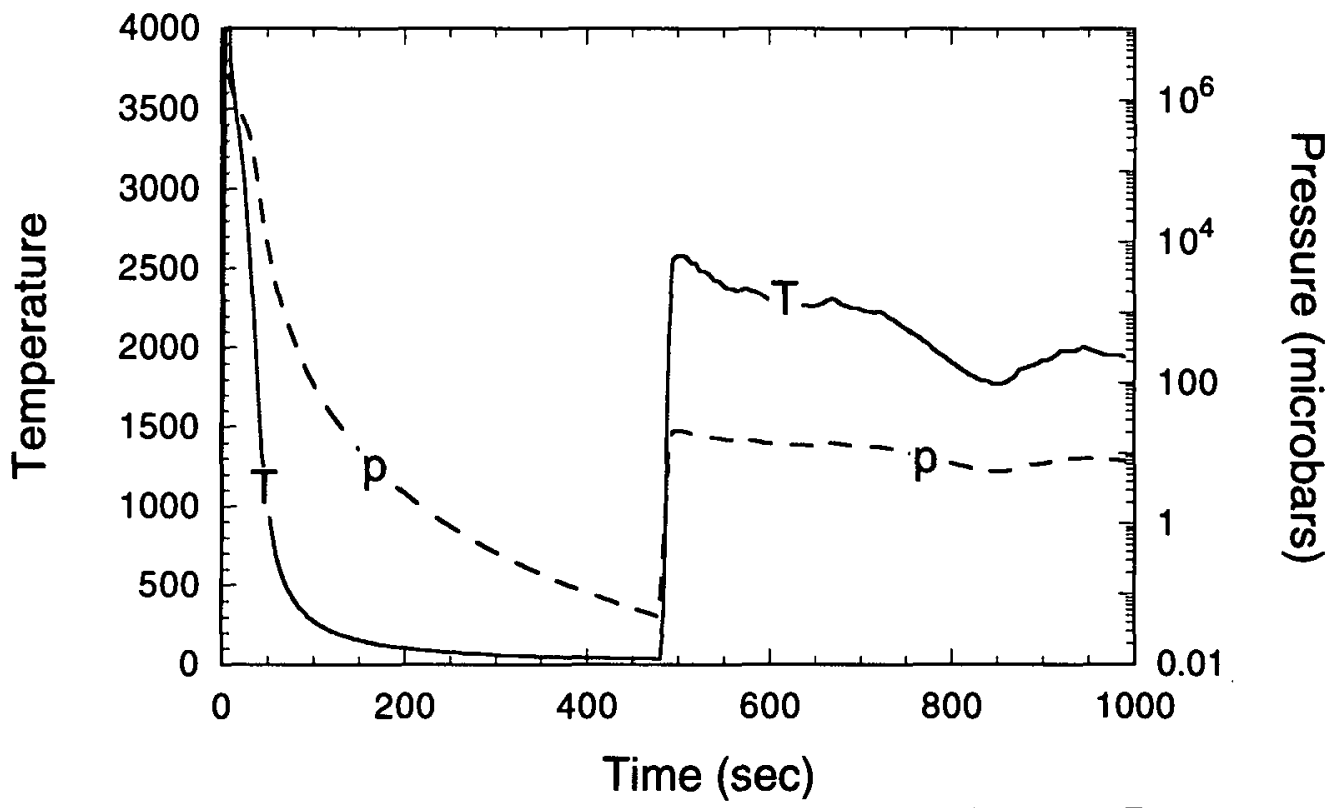

Figure 12. Temperature and pressure history of the parcel shown in Fig. 7.

rium model is that in a kinetics model every chemical reaction must be specified. If no favorable reaction exists, a thermochemically favored species need not form, and the system can settle into a metastable disequilibrium. There are two sorts of kinetic inhibition. One occurs when there really is no favorable reaction. This often happens in a rapidly cooled parcel, because many chemical reactions become very slow at low temperatures. Such a system would be described as quenched. The other, unique to models, occurs when important favorable reactions are unknown, unguessed, or omitted. As relatively few reactions have been accurately measured and tabulated, the modeller needs to invent a great many plausible reactions that, if neglected, would leave an incomplete system producing skewed results. As tables of measured reaction rates are at best incomplete, a great many reactions are guesswork, and the detailed results must be regarded with caution. Thermochemical equilibria calculations are relatively immune from these problems, but their relevance to disequilibrium systems is questionable.

A good, albeit sensitive, example of the limitations of a kinetics model is my previous conclusion that $\mathrm{S}_{2}$ would be the major sulfur species in strongly shocked dry jovian air, rather than CS, as predicted by thermochemical equilibrium (Zahnle et al. 1995). This result now appears to be more wrong than right. The missing pathway to CS was $\mathrm{CH}_{3}+$ $\mathrm{S} \longrightarrow \mathrm{H}_{2} \mathrm{CS}$, an invented, but crucial, reaction. We have since added $\mathrm{HCS}$ and $\mathrm{H}_{2} \mathrm{CS}$ to our list of species calculated; these additions are important as they provide the main channel for $\mathrm{CS}$ and $\mathrm{CS}_{2}$ formation. We now find that $\mathrm{CS}$ and $\mathrm{CS}_{2}$ are usually the major products of sulfur shock chemistry in dry jovian air, as predicted by thermochemical equilibrium.

Thus, again, we must address the presence of $S_{2}$ as a major product. Our earlier finding that $\mathrm{S}_{2}$ can be a disequilibrium byproduct of hydrogen recombination in the low pressure reentry shock (Zahnle et al. 1995) remains valid for a range of chemical compositions. Fig. 13 follows the chemistry of a strongly shocked parcel of plume gas (the parcel's temperature and pressure follow Fig. 12). The parcel's composition is assumed to be $50 \%$ cometary, $50 \%$ jovian air by mass. "Comet" is here defined as a mixture of $\mathrm{C}_{2} \mathrm{H}_{2}$, $\mathrm{H}_{2} \mathrm{O}, \mathrm{NH}_{3}$, and $\mathrm{H}_{2} \mathrm{~S}$, with $\mathrm{C}, \mathrm{O}, \mathrm{N}$, and $\mathrm{S}$ present in solar proportions. Silicates are 


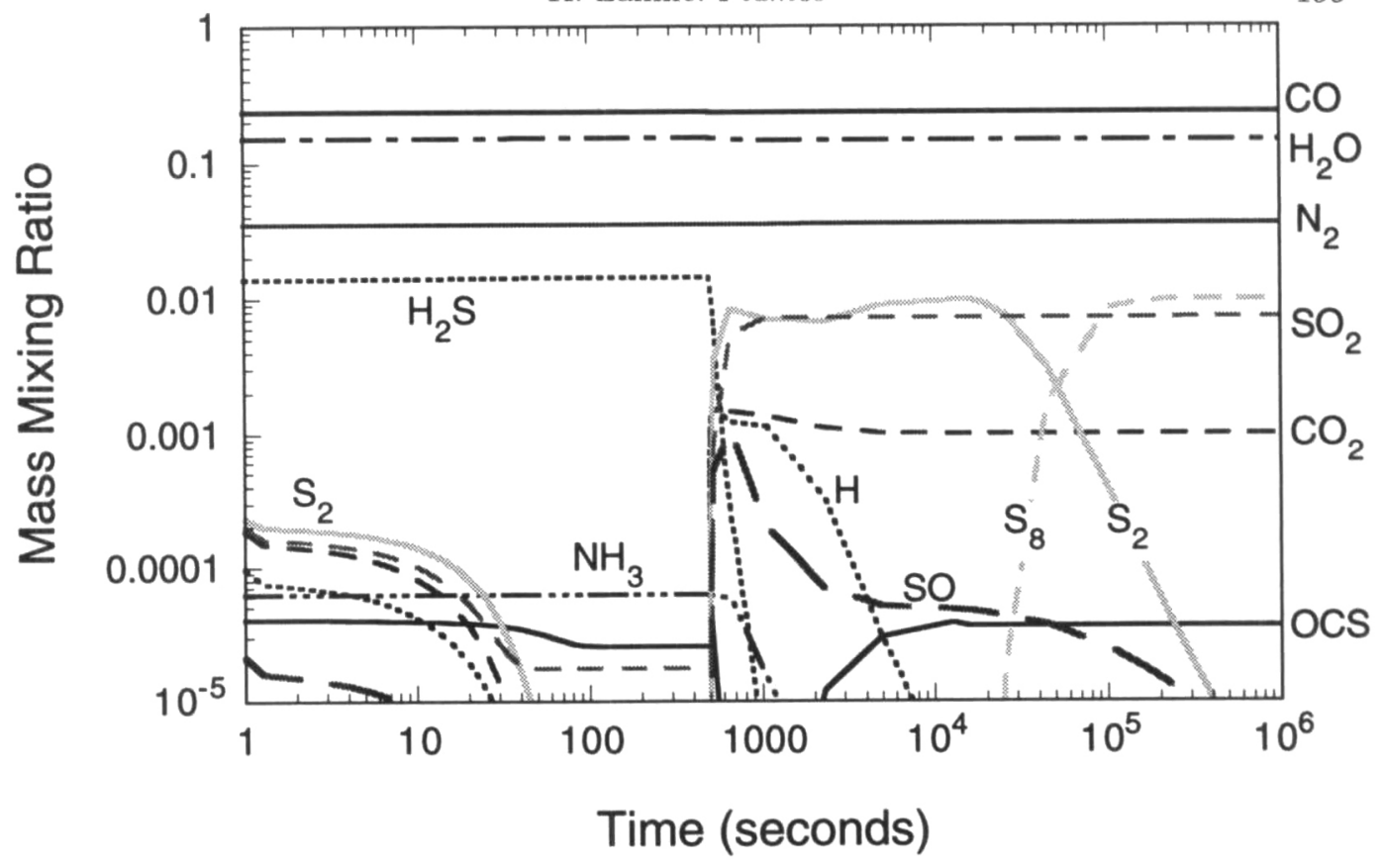

FIgure 13. Time-dependent chemistry in a representive parcel of shocked plume gas. The gas is assumed $50 \%$ comet, $50 \%$ jovian air, by mass. Elemental sulfur is a major product. $\mathrm{H}_{2} \mathrm{~S}$ survives the fireball, but is consumed on reentry. As a solar composition gas is relatively oxidizing, other major products include $\mathrm{SO}_{2}$ and $\mathrm{CO}_{2}$.

ignored-it is assumed that they will reform to stochiometrically equivalent silicates. In this parcel $S_{2}$ is a major sulfur compound after reentry, and remains so while the gas remains warm. Eventually the gas cools to the point where $S_{2}$ polymerizes to $S_{8}$, which probably condenses.

Figure 13 is concerned with the fate of a single parcel shocked to $T^{\prime}=2500 \mathrm{~K}$ and $p^{\prime}=20$ microbars. In this particular parcel, the final sulfur products are $\mathrm{S}_{8}$ and $\mathrm{SO}_{2}$; some OCS forms, as well. As $\mathrm{SO}_{2}$ was not observed to be a major product, this parcel is probably too oxidizing to fit SL9. Other major products of this O-rich gas are $\mathrm{H}_{2}, \mathrm{CO}$, $\mathrm{H}_{2} \mathrm{O}, \mathrm{N}_{2}$, and $\mathrm{CO}_{2}$, the latter also unreported.

Figures 14 and 15 generalize results from parcels like that shown in Fig. 13 to a range of different peak shock temperatures and pressures. They show the final products in the parcel after the parcel has cooled. These figures are prepared for dry and wet jovian air (i.e., comet-free). The peak shock temperature in the fireball is assumed to be twice $T^{\prime}$ (i.e., $v_{z}^{2} \approx v^{2} / 2$ ). For specificity we use Eq. 4.7 with $\eta=0.5$ to relate $T^{\prime}$ to $p^{\prime}$. The different $T^{\prime}$ correspond to a range of different reentry velocities, which in turn correspond to a range of distances $\left(r \sim 2 v_{z}^{2} / g\right)$ from the impact site; approximate distances are also indicated.

The products of shocked dry jovian air (Fig. 14) are of bewildering complexity. For shocks in the range $2000<T^{\prime}<2500 \mathrm{~K}$, especially complex products are formed from pieces of $\mathrm{CH}_{4}$ and $\mathrm{NH}_{3}$. The products shown here, $\mathrm{C}_{2} \mathrm{H}_{n}, \mathrm{C}_{4} \mathrm{H}_{2}$, and $\mathrm{HCN}$ are just the simplest; a wide-range of more complicated hydrocarbons, nitriles, and amines are to be expected in nature. At higher shock temperatures the products are simpler, with $\mathrm{N}_{2}$ favored among nitrogen compounds and $\mathrm{C}_{2} \mathrm{H}_{2}$ favored among the hydrocarbons. In reality it is probable that much of the carbon we assign to $\mathrm{C}_{2} \mathrm{H}_{2}, \mathrm{C}_{2} \mathrm{H}_{4}$, and $\mathrm{C}_{4} \mathrm{H}_{2}$ actually ends up in more complicated compounds or particulates that would qualify as 


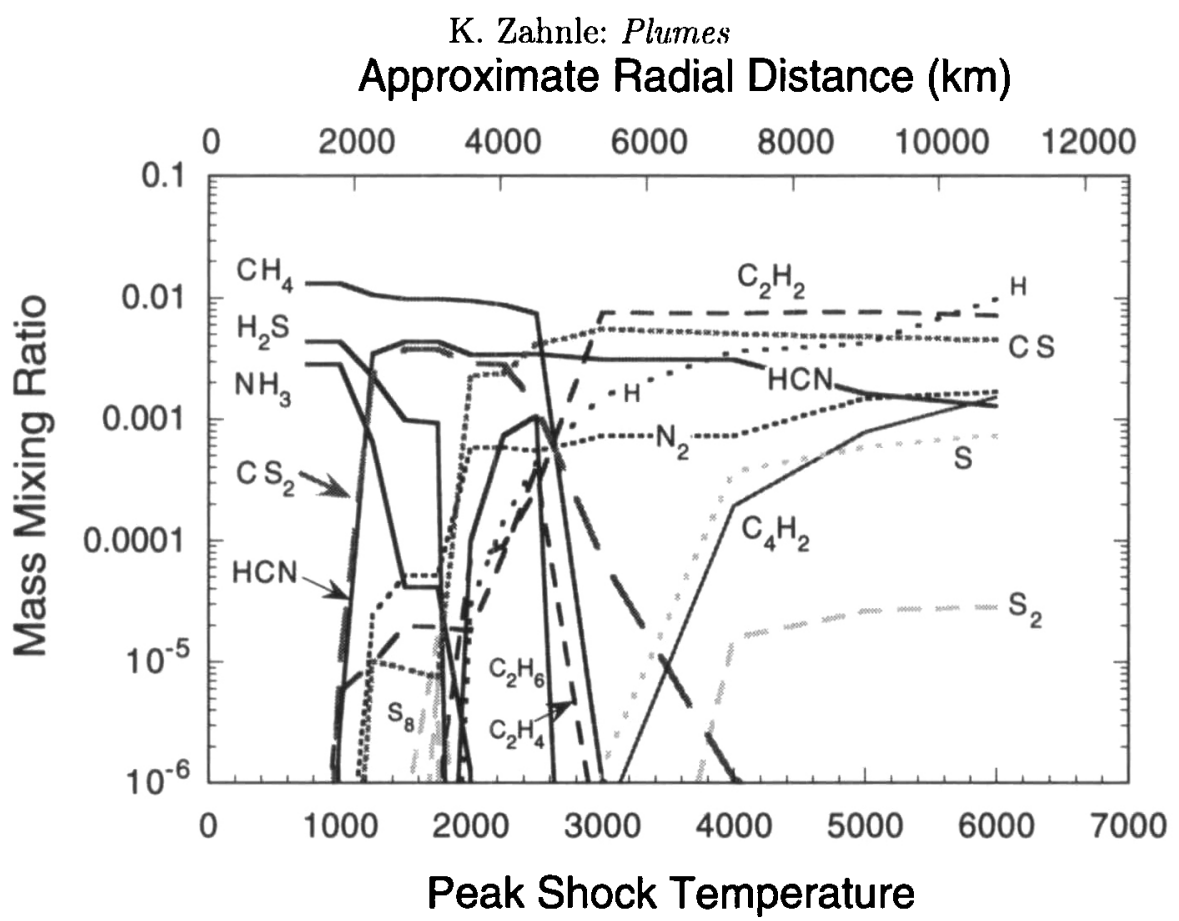

FIGURE 14. Reentry products in dry jovian air as a function of peak shock temperature. Also shown is the corresponding distance from the impact site. This C-rich gas produces a bewildering array of chemical products, but in this case neither $S_{2}$ nor $S_{8}$ are prominent among them. Products for $T^{\prime}<2000 \mathrm{~K}$ are mostly determined by quenching of the fireball rather than the reentry shock.

a kind of tholin; HCN may have the same fate. Whether CS or $\mathrm{S}_{n}$ forms depends on the availability of reactive carbon. If most $C$ goes into refractory grains, $S_{n}$ would be more favored than shown here. The same applies to $\mathrm{HCN}$ and $\mathrm{N}_{2} ; \mathrm{N}_{2}$ is more favored if $\mathrm{C}$ gets tied up in grains. $\mathrm{CS}$ is also known to polymerize readily, even explosively (Moltzen et al. 1988), so it too may have been quickly incorporated into grains. Note that carbonaceous particulates would not be expected to form for peak shock temperatures less than about $2000 \mathrm{~K}$, which requires reentry velocities greater than about $4 \mathrm{~km} \mathrm{~s}^{-1}$.

Figure 15 shows reentry products in wet jovian air as a function of peak shock temperature. Here we do find elemental sulfur as a major product, especially for $2000<T^{\prime}<$ $3000 \mathrm{~K}$, while at higher temperatures $\mathrm{SO}_{2}$ supplants it. At very high $T^{\prime}, \mathrm{CO}_{2}$ and even $\mathrm{O}_{2}$ become major products. (Recall that $T^{\prime}$ is the peak shock temperature, which is not the same as the temperature when these products form.) Under other circumstances (not shown here), shocked wet jovian air can produce a range of reduced products, principally $\mathrm{CS}, \mathrm{CS}_{2}$, and $\mathrm{HCN}$-these form if the pressures are high enough and the temperatures are in the narrow range $(\sim 2000 \mathrm{~K})$ where $\mathrm{CH}_{4}$ and $\mathrm{H}_{2} \mathrm{~S}$ react but $\mathrm{H}_{2} \mathrm{O}$ does not. Thus shock production of tholins from jovian air is not necessarily precluded by the presence of abundant water.

Figure 16 gives the final sulfur products of a shocked $10^{14} \mathrm{~g}$ comet mixed with an equal mass of jovian air, shown as functions of the $\mathrm{C} / \mathrm{O}$ ratio in the reacting gas. The total chemical product is computed by summing the products of all shock temperatures, weighted by the mass of gas shocked to that temperature. When taken at face value, the report that the $\mathrm{K}$ impact produced $2.5 \times 10^{14} \mathrm{~g}$ of $\mathrm{CO}$ (Lellouch, this volume) would imply a $\mathrm{K}$ fragment mass as high as $5 \times 10^{14} \mathrm{~g}$; this mass is about two or three times larger than that obtained from the infrared light curve. 


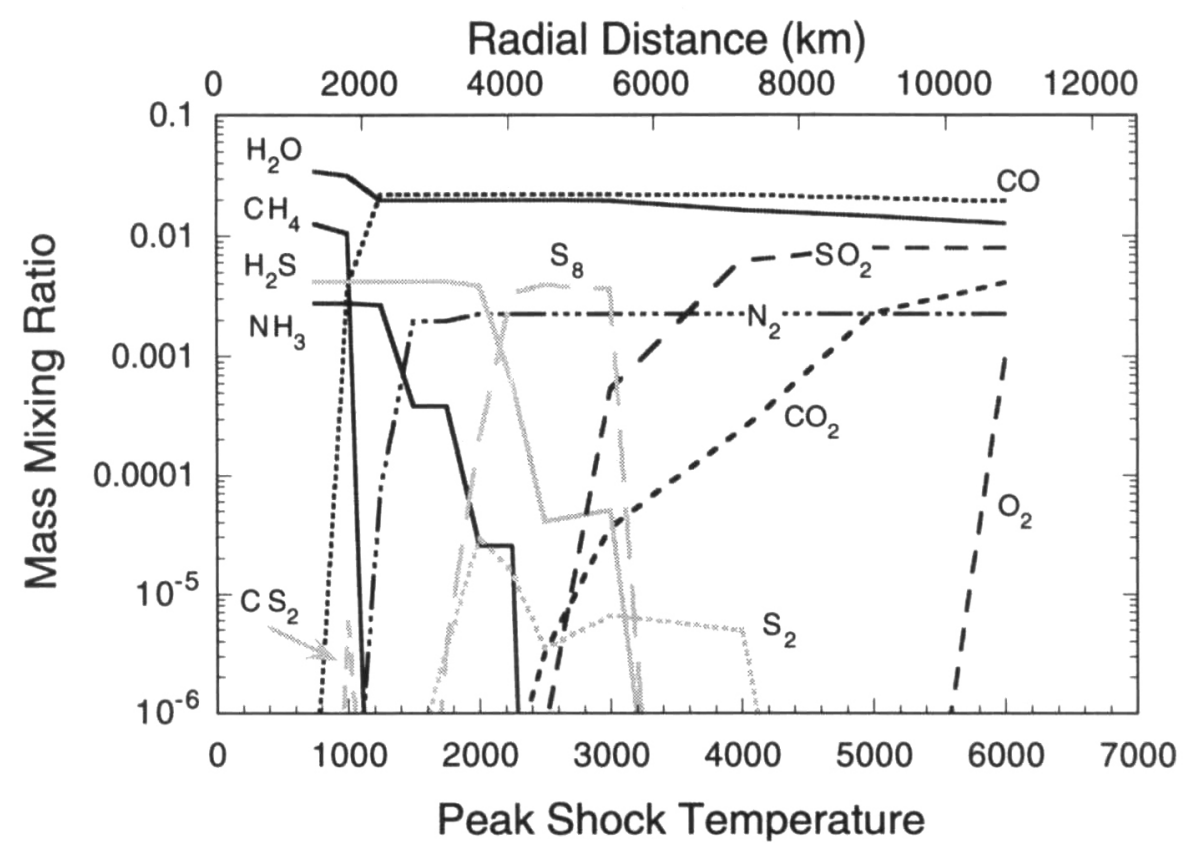

FIgURE 15. Reentry products in wet jovian air as a function of peak shock temperature. Stairstepping by $\mathrm{NH}_{3}$ and $\mathrm{H}_{2} \mathrm{~S}$ is an artifact. Products for $T^{\prime}<2000 \mathrm{~K}$ are mostly determined by quenching of the fireball rather than the reentry shock. Note that elemental sulfur is favored by middling shock temperatures, while the highly oxidized products are favored by high shock temperatures.

Sulfur compounds provide a measure of the oxidation state of the shocked gas. Where $\mathrm{O}>\mathrm{C}$, the primary products are $\mathrm{SO}, \mathrm{SO}_{2}$, and $\mathrm{S}_{n}$. Where $\mathrm{C}>\mathrm{O}$, the products are $\mathrm{CS}$, $\mathrm{CS}_{2}, \mathrm{H}_{2} \mathrm{~S}$, and $\mathrm{S}_{n}$. Interestingly, in these calculations OCS appears to be an indicator of an oxidized gas, but this result could well be model-dependent. Elemental sulfur is exceptional in that it forms at all $\mathrm{O} / \mathrm{C}$ ratios. Results are qualitatively similar for reentry shock chemistry of wet jovian air, as can be inferred by comparing Figs. 14 (dry jovian air) and Fig. 15 (wet jovian air) to Fig. 16. Addition of jovian $\mathrm{H}_{2}$ favors $\mathrm{S}_{8}$ at high $\mathrm{O} / \mathrm{C}$ and disfavors $\mathrm{S}_{8}$ at low $\mathrm{O} / \mathrm{C}$; and of course favors $\mathrm{H}_{2} \mathrm{~S}$ generally.

At this point it no longer appears that chemistry by itself can distinguish between shallow and deep explosions. The redox state of the reactants is ambiguous, and in any event most of the $\mathrm{CO}, \mathrm{H}_{2} \mathrm{O}$, and S-species probably derive from the comet. The early idea that much of the observed sulfur was jovian was driven by a perceived need to produce $10^{15} \mathrm{~g}$ of $S_{2}$. This need has vanished as the estimates for the mass of $S_{2}$ at the impact sites have been revised downward.

Despite the presence of abundant $\mathrm{H}_{2} \mathrm{O}$, there is no evidence that the reacting gases were O-rich. On the contrary, neither $\mathrm{SO}_{2}$ nor $\mathrm{CO}_{2}$ appear to have been unambiguously detected, while CS, $\mathrm{CS}_{2}$, and $\mathrm{HCN}$ were prominent products. There is also the possibility that the dust is carbonaceous. On the other hand, the inferred high abundance of $S_{2}$ is consistent with a mildly oxidized composition, and the presence of abundant $\mathrm{H}_{2} \mathrm{O}$ would appear to require $\mathrm{O}>\mathrm{C}$.

Hot water and $\mathrm{CO}$ are almost certainly cometary. Excavation of modest amounts of jovian water requires that the water be lifted from within the clouds, where the water mixing ratio is limited by the saturation vapor pressure, and would therefore have a very small scale height, on the order of 2 or $3 \mathrm{~km}$. The amount of water excavated would 


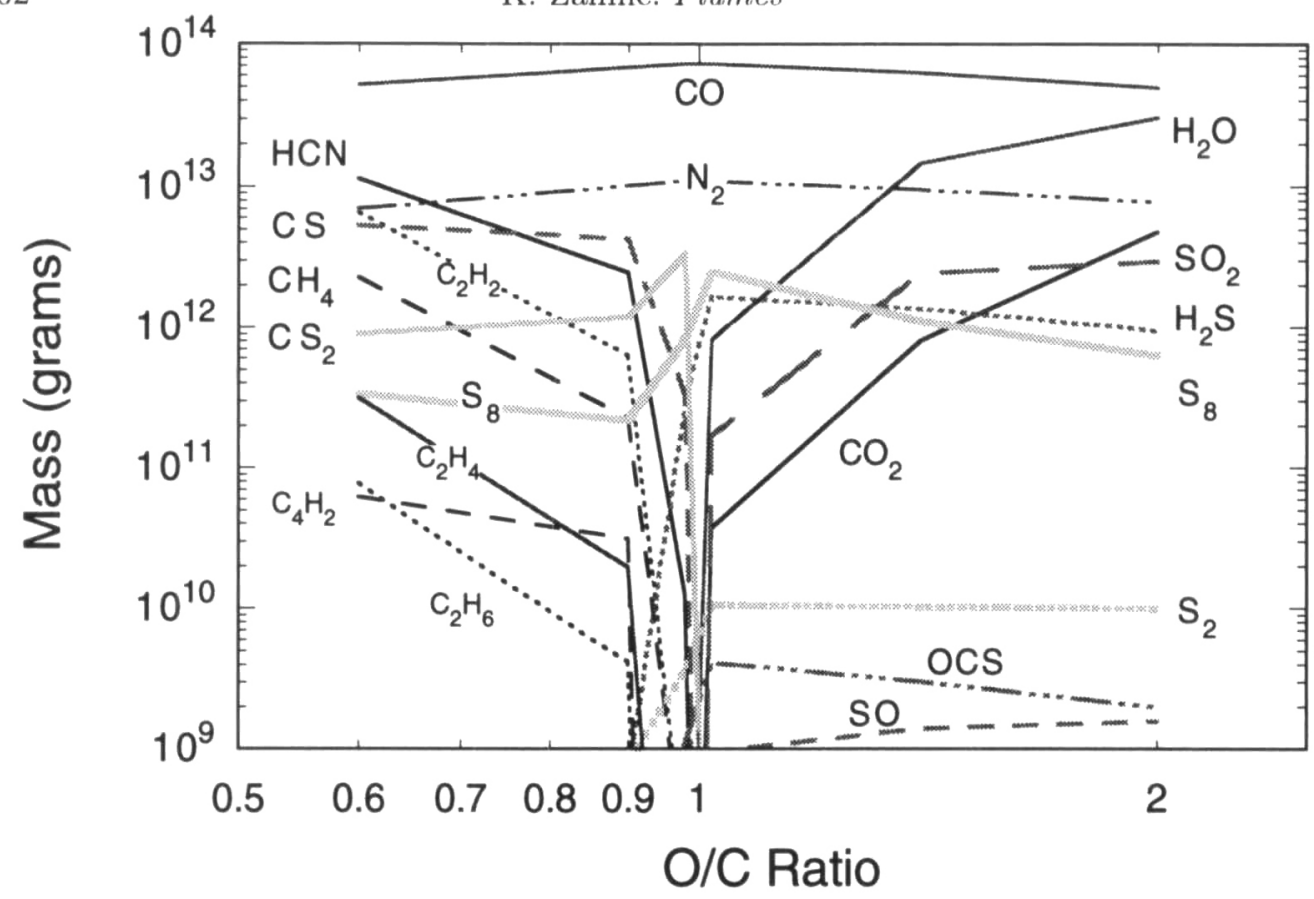

FIGURE 16. Total product of a plume $50 \%$ air, $50 \%$ comet by mass, produced by a $10^{14} \mathrm{~g}$ comet, as a function of the comet's $\mathrm{C} / \mathrm{O}$ ratio. It is assumed that silicates form grains that do not participate in the chemistry. The product is averaged over reentry shocks from $1500 \mathrm{~K}$ to $5000 \mathrm{~K}$. The chemical products are not indicative of any particular pressure or temperature. But is also plain that no single $\mathrm{O} / \mathrm{C}$ ratio can explain the full range of products seen in the aftermath of SL9.

necessarily vary drastically from impact to impact; its roughly comparable presence following the large $\mathrm{K}$ impact and the smaller $\mathrm{R}$ impact (Sprague et al. 1996; Bjoraker et al. 1995) cannot be understood as jovian. An analogous argument should apply to nitrogen and sulfur species if the explosions took place within the putative $\mathrm{NH}_{4} \mathrm{SH}$ clouds; I am unaware of evidence that argues either way. Nonetheless, as Fig. 14 shows, shocked dry jovian air offers a good potential source of $\mathrm{HCN}, \mathrm{CS}_{2}$, and $\mathrm{CS}$ that might complement the more oxidized products of the more highly shocked, comet-fouled plume.

An intriguing possibility is that water condensed in the plume and that the ice grains passed through the rarefied gases of the reentry shock intact, only to flame out as meteors a few seconds later at somewhat higher pressures, in a gas that was more purely jovian. Here the $\mathrm{O} / \mathrm{C}$ ratio may have been locally high, or temperatures too low for $\mathrm{H}_{2} \mathrm{O}$ to react. Such a scenario could physically separate reacting sulfur from oxygen, and might also leave the reacting gas in the shock oxygen-depleted, thereby producing two redox regimes from the same O-rich cometary ejecta. And if both $\mathrm{C}$-rich grains and O-rich ice failed to fully vaporize in the shock, the odds in favor of $S_{2}$ are raised dramatically.

\section{Rings}

One of the more intriguing features observed by HST were rings, encircling several of the impact sites, that expanded radially at constant velocities of order $450-500 \mathrm{~m} \mathrm{~s}^{-1}$ (Hammel et al. 1995). The rings moved too slowly to be shock waves or acoustic waves. Ingersoll \& Kanamori (1995) suggest that the rings are stratospheric manifestations of gravity waves propagating in a wet troposphere. In their model the speed of the wave is 
set by the static stability of the troposphere, which in turn depends on its water content. Conventional Jupiters are constructed with 2-3 times solar water, for which Ingersoll \& Kanamori (IK) calculate leisurely gravity wave speeds of order $150 \mathrm{~m} \mathrm{~s}^{-1}$. To bring the velocity up to the observed $450 \mathrm{~m} \mathrm{~s}^{-1}$ requires raising the water content to 10 times solar. The model predicts that all impacts would produce waves of the same speed (as observed), and because the waves are linear they do not slow down (as observed).

A weakness of the IK model is that it doesn't actually account for the ring. There are two problems. The first is that the ring itself appears to be high in the stratosphere and, judging by its color, its composition is not obviously different from that of other impact debris. In the IK model the ring would be a condensation cloud, but what is the condensible? The obvious candidates, water and ammonia, are white, while a suite of complex organics would not in general be expected to condense and subsequently evaporate with anything like a well-defined phase change. Perhaps the most viable candidate is molecular sulfur $\left(S_{8}\right)$, but here again its color works against it.

A second argument against a condensation cloud is more telling: where are the sound waves? Strong acoustic waves moving at velocities of order $1 \mathrm{~km} \mathrm{~s}^{-1}$ are inevitable. Strong stratospheric gravity waves with velocities of order $900 \mathrm{~m} \mathrm{~s}^{-1}$ are also generated (IK suggest that the explosions were below 10 bars to minimize stratospheric waves). These waves quickly develop into shock waves with strong temperature and pressure amplitudes. We see these waves in abundance in our numerical simulations. Condensibles would respond to these $1 \mathrm{~km} \mathrm{~s}^{-1}$ waves at least as strongly as they would to the distant pulse of a tropospheric gravity wave. That $1 \mathrm{~km} \mathrm{~s}^{-1}$ acoustic waves were not seen seems clear, and hence it is difficult to accept the hypothesis that the $450 \mathrm{~m} \mathrm{~s}^{-1}$ ring marks the passage of a wave through a condensible material.

An alternative hypothesis is that the rings are nonlinear stratospheric gravity waves. Breaking waves can sweep material along with them. We have suggested that the rings are actual rings of impact debris flowing outward from the impact site (Young et al. 1995). This hypothesis is suggested to us by the numerical models, in which we see just such a ring of airburst debris propagating outwards at $500 \mathrm{~m} \mathrm{~s}^{-1}$ at the base of the stratosphere. (Fig. 17 and 18).

The numerical ring is an attractive candidate for several reasons. It has the right velocity. It has the right location, centered on the stratospheric waveguide at 10-50 mbar. Its velocity happens to be the same as the critical velocity $u=N H\left(490 \mathrm{~m} \mathrm{~s}^{-1}\right.$ in our numerical model), where $N$ is the Brunt-Väisäla frequency; i.e., the numerical ring appears to propagate at a Froude number of 1 . It is far from clear that this is more than simple coincidence, but it is an intriguing coincidence, nonetheless.

But as IK point out, the nonlinear wave has a severe disadvantage: it slows down. Our numerical wave illustrates precisely this defect. After maintaining a constant velocity of $490 \mathrm{~m} \mathrm{~s}^{-1}$ for $700 \mathrm{~s}$, it slows. The real rings lasted at least $10^{4} \mathrm{~s}$. Numerical experiments indicate that the failure to propagate is not a numerical effect. The energy of the numerical wave is only about $10^{25} \mathrm{crgs}$; i.e., only about $1 \%$ of the impact energy. Numerical experiments indicate that doubling the energy in the wave doubles the area it covers. Hence we seek an additional energy source.

A possible source of energy is the latent heat of condensation of water vapor lifted by the impact. Stoker (1986) shows that, because it is dense, moist jovian air tends to be stable against convection, but if lifted by more than about $7 \mathrm{~km}$ the cumulative effects of condensation (heating, water depletion) are sufficient to cause the parcel to rise rapidly and indefinitely.

The explosion leaves a transient crater in the atmosphere. Air rises from below to fill the hole. This can be seen in the lowest lines of tracer particles in Fig. 17. The 


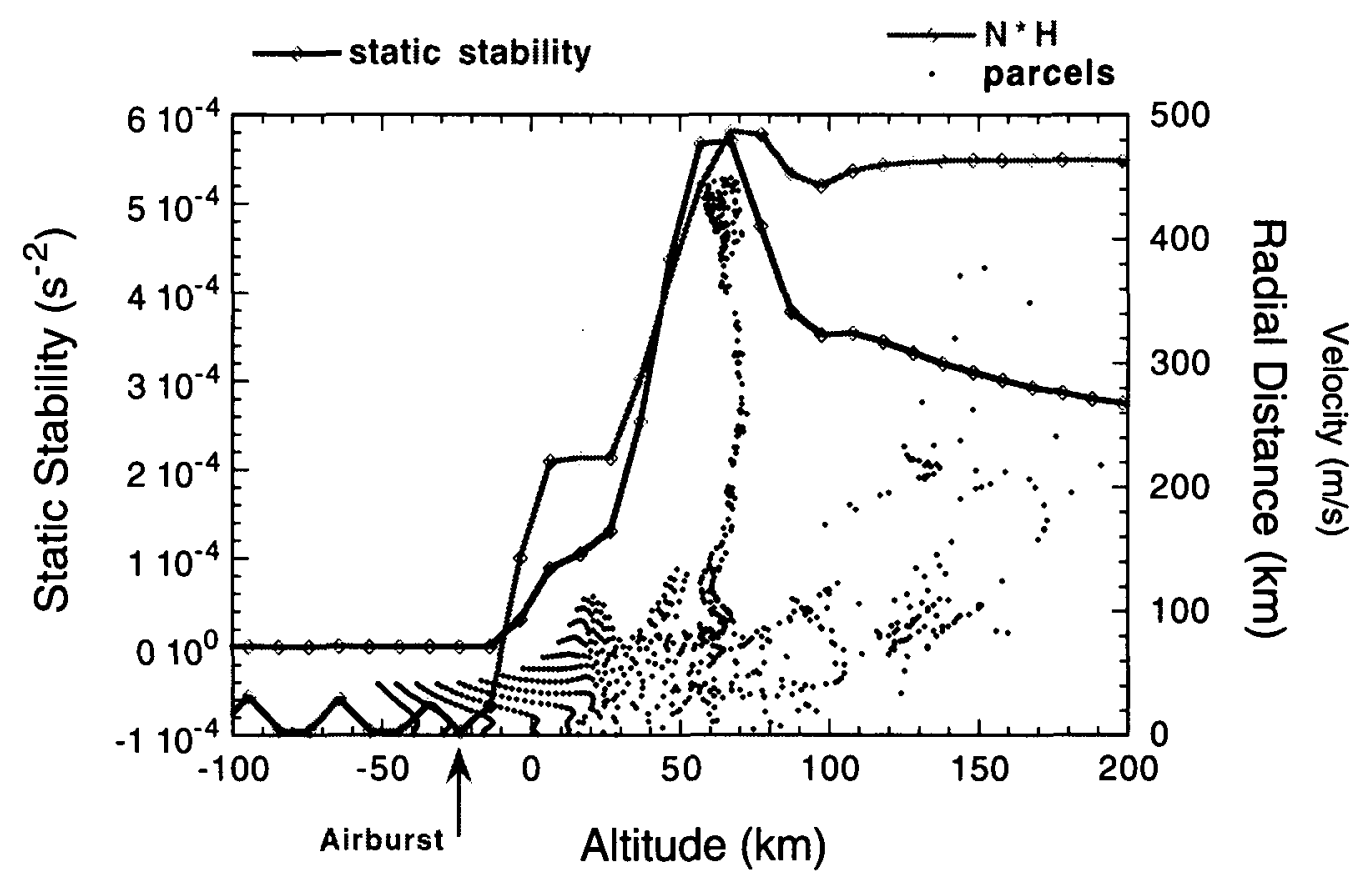

FIGURE 17. This figure shows the locations of several hundred tracer particles $950 \mathrm{~s}$ after impact in the general vicinity of the impact site, as calculated by ZEUS-3D. The particles were initially packed in a cylinder $50 \mathrm{~km}$ radius and extending in $z$ from $-65 \mathrm{~km}$ to $+100 \mathrm{~km}$, where $z=0$ at 1 bar. The explosion released $2 \times 10^{27}$ ergs at $z=-25 \mathrm{~km}$. Many of the most strongly shocked particles are thousands of kilometers distant, well beyond the frame of this picture. The feature at $z=65 \mathrm{~km}$ and $r=450 \mathrm{~km}$ is a ring of moderately strongly shocked jovian air driven from deep inside the original cylinder. We also show the static stability of the jovian atmosphere that was used in this numerical simulation. It is interesting to note that the numerical ring travels within the waveguide defined by the static stability. The velocity of the ring is shown in Fig. 18.

lowest line of parcels began at $z=-65 \mathrm{~km}$. In the numerical experiment they are lifted $25 \mathrm{~km}$ along the $z$-axis, and about half as much at $r=50 \mathrm{~km}$. These parcels were probably water-rich when they began to rise, but on being lifted $>10 \mathrm{~km}$ effectively all the water they carry condenses, with concomitant release of latent heat of condensation. The amount of energy available in latent heat can rival that released by the impact itself.

As a specific example, assume that jovian water is enhanced 5 -fold over cosmic. The corresponding lifting condensation level would be at 8.5 bars and $308 \mathrm{~K}(z \approx-75) \mathrm{km}$. The available latent heat of condensation in a cylinder of this air $50 \mathrm{~km}$ radius and $30 \mathrm{~km}$ thick is $\sim 2 \times 10^{26} \mathrm{ergs}$. These dimensions are in all likelihood smaller than the actual volume of lifted moist air, and hence this estimate is, in all likelihood, smaller than the actual amount of energy that would be available to the convective plume. This energy is mostly delivered to the base of the stratosphere, where the plume spreads as a large but otherwise conventional anvil (Note that the anvil, because it is cold, would contain very little water. The water that fueled the plume falls out deep in the troposphere.) It is this additional energy that we speculate powers the ring, and keeps it going well beyond the predictions of early numerical experiments. 


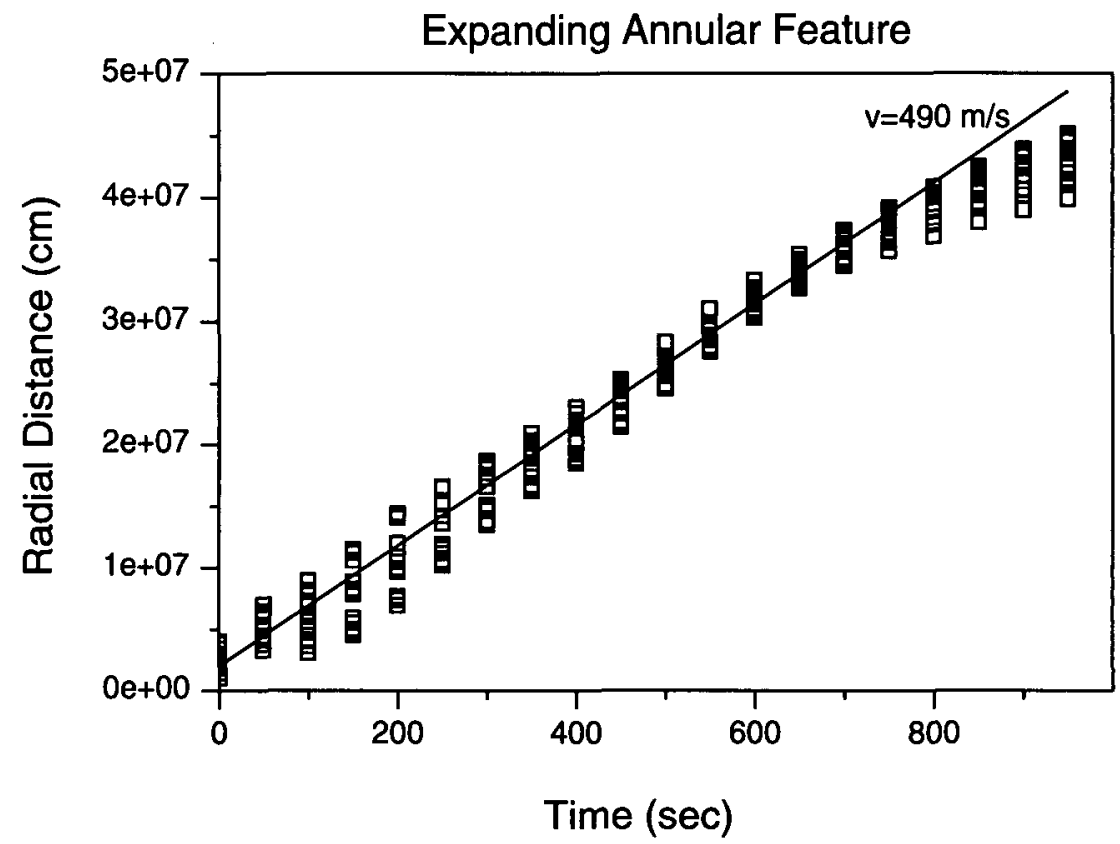

FIgURE 18. The expansion velocity as a function of time of the annular feature shown in Fig. 17, as defined by 13 representative tracer particles. The ring maintains a steady velocity of about $490 \mathrm{~m} \mathrm{~s}^{-1}$ for some $700 \mathrm{sec}$, although individual particles cycle through slower and faster velocities. This is a breaking wave. After $700 \mathrm{~s}$ it slows down, and the wave ceases to break.

A third interesting possibility is a hybrid model, in which the master wave is a linear disturbance in the wet troposphere, but which manifests itself as a nonlinear, breaking wave in the stratosphere, where the amplitude of the wave might well be larger than in the troposphere. In the hybrid model the tropospheric wave serves both as energy source and pacemaker, while the visible wave remains a material ring of impact debris and/or charred jovian air travelling along the stratospheric waveguide.

\section{Plume heights}

Several of the SL9 ejecta plumes were observed by the HST to reach approximately the same height, about $3000 \mathrm{~km}$ above the jovian cloudtops (Hammel et al. 1995). The duration of the infrared events, produced by the plume falling back on the atmosphere, measures time aloft and hence provides a second, more sensitive measure of plume height. The light curves (Nicholson, this volume) indicate that the largest impacts produced modestly higher plumes (compare $\mathrm{K}$ to $\mathrm{D}$ ), but the difference was not large. Evidently all the plumes were launched with about the same vertical velocity, roughly $10-13 \mathrm{~km} \mathrm{~s}^{-1}$. As the impactors themselves were not all the same, nor the impacts equally luminous, nor the plumes equally opaque, the similar plume heights has been seen as a puzzle needing explanation.

Figure 19, which summarizes our explanation, compares loci of constant plume height to calculated airburst altitudes for impacts of different energy. It is immediately apparent that the curves are approximately parallel. But where do these curves come from?

The characteristic radius $R_{s}$ of a strong point explosion of energy $E_{i}$ in a homogeneous gas with ambient pressure $p_{a}$ is roughly

$$
R_{s} \approx 0.5\left(E_{i} / p_{a}\right)^{1 / 3}
$$




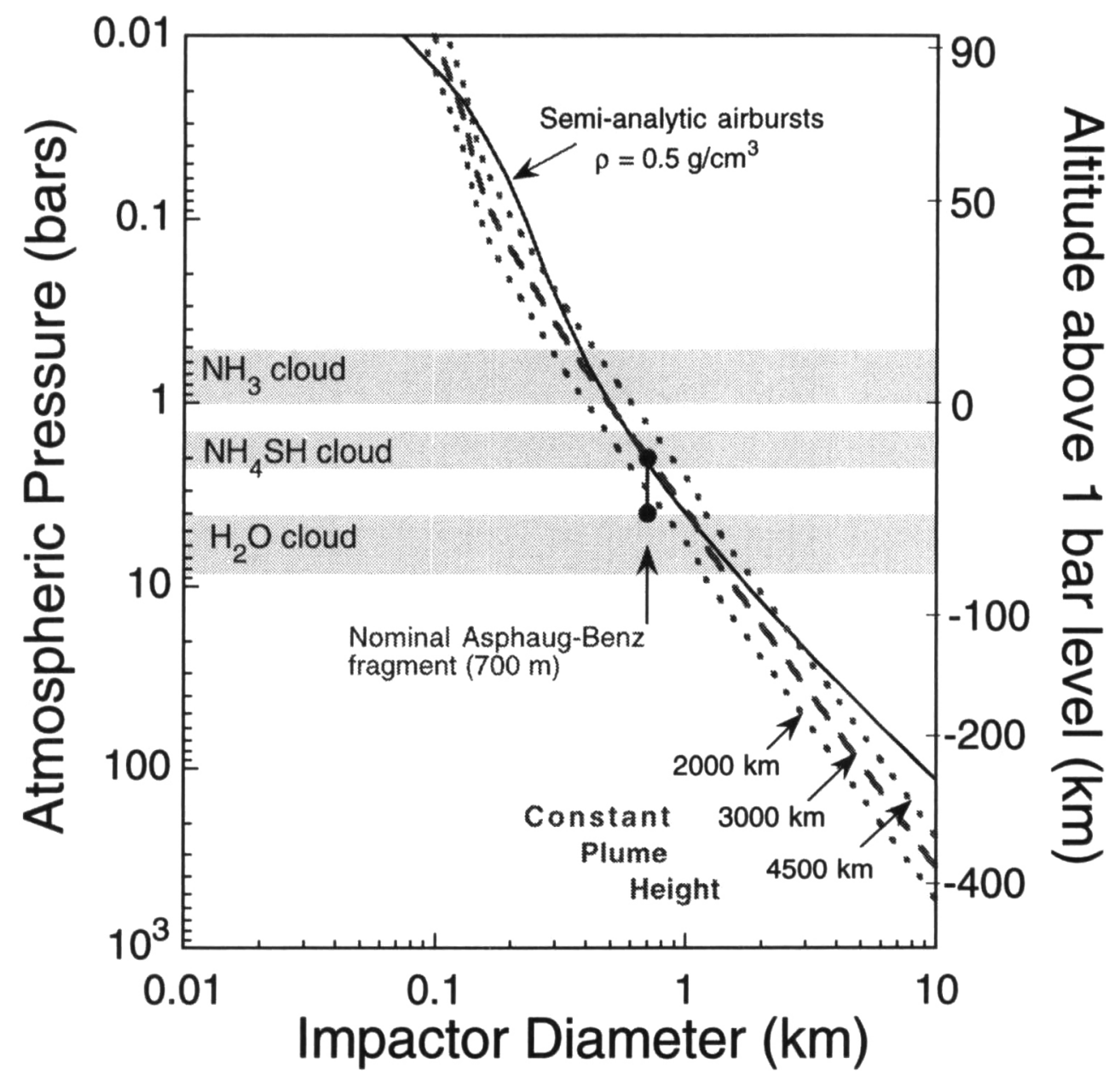

Figure 19. Airburst altitude, defined as the altitude of peak kinetic energy loss, for comets of density $0.5 \mathrm{~g} \mathrm{~cm}^{3}$, is shown by the solid curve. The intermittent curves show loci of constant plume height. The labels, in kilometers, give relative plume heights. We assume that the airburst curve is correct, and use the observed $3000 \mathrm{~km}$ plume height to calibrate the curves of constant plume height. The diameter of the largest fragment, consistent with the tidal breakup analysis of Asphaug \& Benz (1994), is marked by a dumbbell. The top of the dumbbell is placed on the airburst curve. As material can continue to move downward after it is shed by the comet, the effective explosion altitude is probably deeper. The bottom of the dumbbell, based on numerical results of Mac Low and Crawford et al., allows for this.

This relation can be obtained by equating the ambient energy within a sphere of radius $R_{s}$ to the energy of the explosion. The characteristic length scale of the atmosphere is its scale height $H$. Where $R_{s} \ll H$ the explosion is spherically symmetric and smothered by the atmosphere. Where $R_{s}$ and $H$ are more nearly equal the explosion is distorted by the gradient in the background atmosphere, and if $R_{s}>H$ the explosion is expected to blow out into space. Accordingly, one expects the dimensionless ratio $R_{s} / H$, or the functionally equivalent dimensionless ratio $E_{i} / p_{a} H^{3}$, to determine the shape of the transient crater and the shape of the plume, and in particular its opening angle.

We showed elsewhere (Zahnle \& Mac Low 1994) that ejecta velocities should scale as $\sqrt{E_{i} / \rho_{a} H^{3}}$. This is agreeable with $R_{s} / H$ being the key dimensionless parameter, because $\sqrt{E_{i} / \rho H^{3}} \propto c_{s}\left(R_{s} / H\right)^{3 / 2}$, where the sound speed $c_{s}$ is the natural velocity in 
the problem. In its most reduced form, the argument compares the energy released by the explosion to the kinetic energy of the entrained air. As a simple example, assume that the explosion is isotropic, with energy $E_{i} / 4 \pi$ per steradian. The mass of air in the cone above the explosion, per unit solid angle, is $\int_{0}^{\infty} \rho_{a} e^{-z / H} r^{2} d r=\rho_{a} H^{3}$. The asymptotic ejection velocity is therefore

$$
v=\sqrt{\frac{E_{i}}{4 \pi \rho_{a} H^{3}}}=\sqrt{\frac{E_{i} g}{4 \pi p_{a} H^{2}}}
$$

Although Eq. 7.11 gives the right dimensional form, the velocity it predicts is much too slow. For what we have taken to be typical SL9 parameters $\left(E_{i}=2 \times 10^{27} \mathrm{ergs}\right.$, $p_{a}=2$ bars), Eq. 7.11 gives $v \approx 2 \mathrm{~km} \mathrm{~s}^{-1}$, which is much less than the $\sim 10 \mathrm{~km} \mathrm{~s}^{-1}$ produced by numerical simulation (e.g., Fig. 3-6) of the same event. In our 1994 paper, we suggested that the analytic argument fails to get the velocity right because it does not allow for the low density and high temperature of the wake (the wake provides a highway to space); other possibilities are that the assumption of an isotropic explosion is grossly in error, or that the assumption of radial trajectories is badly violated.

Given that ejecta velocities scale as $\sqrt{E_{i} / p_{a} H^{2}}$, the plume height $z_{\max }$ goes as

$$
z_{\max } \approx \frac{v_{\max }^{2}}{2 g} \propto \frac{E_{i}}{p_{a} H^{2}}
$$

where we have identified $z_{\max }$ with $v_{\max }$, the maximum velocity in the toy plume. The curve labeled "constant plume height" in Fig. 19 traces $p \propto E_{i} / H^{2}$.

Airburst altitudes are calculated using the simple semi-analytic model for the deceleration and destruction of large meteoroids presented by Chyba et al. (1993) and Zahnle \& Mac Low (1994). In brief, we numerically integrate standard equations for drag and ablation, supplemented by our nonstandard equation for the effective cross-section. The equations are cast in terms of altitude $z$ and entry angle $\theta$, measured from the zenith. The drag force is

$$
m \frac{d v}{d z}=\frac{1}{2} C_{\mathrm{D}} S \rho_{a} v \sec \theta
$$

where $S$ is the effective cross-section, $\rho_{a}$ is the atmospheric density, and $C_{\mathrm{D}}$ is the drag coefficient ( $C_{\mathrm{D}} \approx 0.9$ for a sphere). Mass loss by ablation is given by

$$
Q \frac{d m}{d z}=\frac{1}{2} C_{\mathrm{H}} S \rho_{a} v^{2} \sec \theta
$$

where $Q$ is a characteristic latent heat of ablation, which we identify with the heat of vaporization $\left(Q \approx 2.5 \times 10^{10} \mathrm{ergs}^{-1}\right.$ for ice), and $C_{\mathrm{H}}(\leq 1)$ is the heat transfer coefficient. Radiative ablation, in which the meteor is evaporated by thermal radiation from the hot bow shock, is important for sinall bodies and low $\rho_{a}$, but becomes unimportant for large bodies at high $\rho_{a}$ (Tauber \& Kirk 1976; Biberman et al. 1980; Zahnle 1992; Zahnle \& Mac Low 1994; Chevalier \& Sarazin 1994; Field \& Ferrara 1995). We use

$$
C_{\mathrm{H}}=\min \left(0.1, \frac{2 \sigma T^{4}}{\rho_{a} v^{3}}\right),
$$

where $C_{\mathrm{H}}=0.1$ is typical for terrestrial meteors at high altitudes. The temperature attained by the shocked gas is strongly regulated by thermal ionization to a value of the order of 30,000 K (Biberman et al. 1980; Zahnle \& Mac: Low 1994).

The third, nonstandard equation describes how the shattered impactor spreads in response to aerodynamic forces. Chyba et al. (1993) approximate the impactor's radius 
by

$$
\frac{d^{2} r}{d t^{2}}=\frac{1}{2} \frac{C_{\mathrm{D}} \rho_{a} v^{2}}{\rho_{i}}
$$

Written in terms of altitude $z$, this becomes

$$
r \frac{d^{2} r}{d z^{2}}+\frac{r}{v} \frac{d v}{d z} \frac{d r}{d z}=\frac{C_{\mathrm{D}} \rho_{a}}{2 \rho_{i}} \sec ^{2} \theta
$$

where $\rho_{i}=0.5$ is the density of the comet. Equation 7.17 is solved numerically in concert with Eq. 7.13 and Eq. 7.14 using $S \equiv \pi r^{2}$. The curves labeled by impactor density in Fig. 19 are the loci of maximum energy deposition. As material can continue to move downward after it is shed by the comet, the effective explosion altitude is probably deeper (see chapter by Mac Low), perhaps by as much as a scale height.

As noted at the beginning of this section, what Fig. 19 shows is that, when plotted against impact energy, loci of constant plume height are nearly parallel to the loci of calculated airburst altitudes. What this means is that the impact of SL9 fragments with diameters ranging from 100-1000 $\mathrm{m}$ generated plumes of similar size and shape. Different plumes differ mostly by their optical depth, by plumefall luminosity, and by the pressure level of atmospheric reentry, all of which are proportional to impactor mass. The essential reason that plumes tend to be the same size and shape is that smaller fragments exploded at higher altitudes, and so had less jovian air to lift, while the larger events, penetrating more deeply, spread their energy over a larger mass of jovian air. But the near constancy of $z_{\max }$ also owes something to coincidence: the greater role of radiative ablation in the flight of smaller objects raises explosion altitudes. If the SL9 fragments had been much larger, the three curves would not have been so nearly parallel, and the differences between the largest and smallest event would have been more pronounced.

\section{Conclusions}

Tidal disruption models show that the parent comet was about $1.5 \mathrm{~km}$ diameter and had a density of $\sim 0.6 \pm 0.1 \mathrm{~g} \mathrm{~cm}^{3}$ (Asphaug \& Benz 1994, 1996; Solem 1994). These models are relatively strongly constrained by observations, and they have relatively few free parameters (diameter, density, and rotation). [However, see chapter by Sekanina for an alternative view. Eds.] The diameter of the parent body is directly proportional to the distance between the leading and trailing fragments, i.e., the distance between $\mathrm{A}$ and W. The density of the parent body is determined by the number of fragments, i.e., the number of letters between $A$ and $W$. If the largest fragment is assigned $1 / 8$ of the total mass, the largest fragments were some $700-800 \mathrm{~m}$ across, had mass of $\sim 1 \times 10^{14} \mathrm{~g}$, and released energies of $\sim 2 \times 10^{27} \mathrm{ergs}$.

A first analysis of the infrared light curves agrees with this size. We estimate that the energy of the $R$ impact plume was about $4 \times 10^{26} \mathrm{ergs}$, about half of which is invested in the ejecta plume. Nicholson (this volume) suggests that the $G, K$, and $\mathrm{L}$ events were about five or ten times more luminous than $\mathrm{R}$, which would place them at $\sim 2-5 \times 10^{27}$ ergs. When cast as the diameter of a $0.5 \mathrm{~g} \mathrm{~cm}^{3}$ comet, a spherical $\mathrm{G}$ would have been $750-1000 \mathrm{~m}$ across.

The chemical evidence is ambiguous, but most indications are that $\mathrm{C}>\mathrm{O}$ in the shocked, reacting gas. Telltale signatures of abundant oxygen- $-\mathrm{SO}_{2}, \mathrm{SO}, \mathrm{CO}_{2}, \mathrm{O}_{2}$-were not seen, while signatures of abundant carbon- $\mathrm{CS}, \mathrm{CS}_{2}$, and $\mathrm{HCN}-$ were. On the other hand, abundant $\mathrm{H}_{2} \mathrm{O}$ would appear to require $\mathrm{O}>\mathrm{C}$, and two other observed sulfur species, $\mathrm{S}_{2}$ and OCS, appear to form more easily in a somewhat oxidized gas. Since on general principles one expects the comet to have had a more-or-less cosmic composition, i.e., 
$\mathrm{O}>\mathrm{C}$, the production of $\mathrm{CS}, \mathrm{CS}_{2}$, and $\mathrm{HCN}$ probably requires $\mathrm{C}>\mathrm{O}$ in the shocked jovian air. This in turn implies that even the largest fragments released the bulk of their energy above the jovian water table, in all likelihood above 5 bars. There is no evidence in favor of the proposition that a significant amount of wet jovian air was shocked strongly enough to coax water to react; i.e., wet jovian air saw only temperatures significantly below $2000 \mathrm{~K}$.

A more direct measure of the impactor masses comes from the mass of CO. The reported mass of $\mathrm{CO}$, some $2.5 \times 10^{14} \mathrm{~g}$ for $\mathrm{K}$ (Lellouch, this volume), would imply impact energies as large as $10^{28} \mathrm{ergs}$, i.e., two or three times higher than the energies estimated by other means. The CO measurements are probably more model dependent, and hence more uncertain, than are the estimates made from tidal disruption and infrared radiation, and probably less model dependent than explosion depths estimated from the apparent absence of jovian water.

The simple pancake model places maximum energy deposition by a $700 \mathrm{~m}$ diameter, $0.5 \mathrm{~g} \mathrm{~cm}^{3}$ comet at 2 bars (Fig. 19). The melange-hot, swept up jovian air, plus disintegrating comet-continues downward some distance before it explodes, perhaps getting as deep as 4 bars before it turns around (see chapters by Crawford and Mac Low for more on these matters). Although some of the comet's mass may plunge deeper, little of its energy does. Concord with the observations is as good as could be hoped.

The outstanding argument in favor of a deep explosion is the identification of expanding rings as a condensation wave excited by a deeply seated tropospheric wave. This model requires deep energy deposition solely in order not to excite analogous stratospheric waves that would also be visible as condesation waves, but which are not observed. We offer as a speculative alternative that the expanding rings were a kind of nonlinear debris wave, in which the dark feature is analogous to the flotsam and jetsam carried by water waves. If they are not condensation waves, the perceived need for deep explosions is removed.

In sum, the SL9 impacts appear to have been small and shallow. Tidal disruption calculations and models of the infrared light curves agree on an average diameter of about half a kilometer, with the largest fragments approaching $1 \mathrm{~km}$; the reported mass of $\mathrm{CO}$ fattens the largest fragment to $1.2 \mathrm{~km}$. The chemical evidence implies that the explosions occured in the presence of sulfur but above the jovian water table. The inferred size, density, and the chemical limit on explosion depth are consistent with preimpact predictions made using simple semi-analytic models. The spectacular plumes were spectacular mostly because the explosions were shallow, not because the explosions were large.

I thank, among others, E. Asphaug, B. Bézard, D. Deming, B. Fegley, J. Graham, J. Harrington, A. Ingersoll, T. Johnson, K. Lodders, J. I. Moses, P. Nicholson, K. Noll, M. Norman, I. de Pater, R. Yelle, R. Young, and of course M.-M. Mac Low, the co-author with all the numbers. K.Z. is supported by the NASA Exobiology Program.

\section{REFERENCES}

Ahrens, T., Takata, T., O'Keefe, J. D., \& Orton, G. 1991 Impact of Comet ShoemakerLevy 9 on Jupiter. Geophys. Res. Lett. 21, 1087-1090.

Anders, E. \& Grevesse, N. 1989. Abundances of the elements: meteoritic and solar. Geochim. Cosmochim. Acta 53, 197-214.

Asphaug, E. \& Benz, W. 1994. Density of comet Shoemaker-Levy 9 deduced by modelling breakup of the parent 'rubble pile'. Nature 370, 120-124. 
Asphaug, E. \& Benz, W. 1996. Size, density, and structure of Comet Shoemaker-Levy 9 inferred by the physics of tidal breakup. Icarus (in press).

Boslough, M., Crawford, D., Robinson, A., \& Trucano, T. 1994. Mass and penetration depth of Shoemaker-Levy 9 fragments from time-resolved photometry. Geophys. Res. Lett. 21, 1555-1558.

Boslough, M., Crawford, D., Trucano, T., \& Robinson, A. 1995. Numerical modelling of Shoemaker-Levy 9 impacts as a framework for interpreting observations. Geophys. Res. Lett. 22, 1821-1824.

Carlson, R., Weissman, P., Hui, J., Smythe, W., Baines, K., Johnson, T. V., Drossart, P., Encrenaz, T., Leader, F., \& Mehlman, R. 1994. Galileo NIMS observations of the impact of comet Shoemaker-Levy 9 on Jupiter. EOS Trans. AGU 75, 401.

Carlson, R., Weissman, P., Segura, M., hui, J., Smythe, W., Johnson, T. V., Baines, K., Drossart, P., Encrenaz, T., \& Leader, F. 1995. Galileo infrared observations of the Shoemaker-Levy $9 \mathrm{G}$ impact fireball: A preliminary report. Geophys. Res. Lett. 22, 1557-1560.

Carlson, R., Weissman, P., Hui, J., Smythe, W., Baines, K., Johnson, T. V., Drossart, P., Encrenaz, T., Leader, F., \& Mehlman, R. 1995. Some timing and spectral aspects of the $\mathrm{G}$ and $\mathrm{R}$ collision events as observed by the Galileo near-infrared mapping spectrometer, In Proceedings: European Shoemaker-Levy 9 Conference (ed. R. West). pp. 69-73.

Chapman, C., Merline, W., Klaasen, K., Johnson, T., Heffernan, C., Belton, M., Ingersold, A., \& The Galileo imaging team. 1995. Preliminary results of Galileo direct imaging of SL9 impacts. Geophys. Res. Lett. 22, 1561-1564.

Chevalier, R., \& SARAziN, C. 1994. Explosions of infalling comets in Jupiter's atmosphere. Astrophys. J. 429, 863-875.

Chyba, C., Thomas, P., \& Zahnle, K. 1993. Nature 361, 40-44.

Crawford, D., Boslough, M., Trucano, T., \& Robinson, A. 1994. Numerical simulations of fireball growth and ejecta distribution during Shoemaker-Levy 9 impact on Jupiter. EOS Trans. $A G U \mathbf{7 5}, 404$.

Crawford, D., Boslough, M., Trucano, T., \& Robinson, A. 1994. The impact of comet Shoemaker-Levy 9 on Jupiter. Shock Waves 4, 47-50.

CRISP, D., \& MEADOws, V. 1995. Near-infrared imaging spectroscopy of the impacts of SL9 fragments C, D, G, K, N, R, V, and W with Jupiter. IAU Colloquium 156: The Collision of Comet P/Shoemaker-Levy 9 and Jupiter held at the Space Telescope Science Institute, p. 25.

DE Pater, I. 1991. The Significance of Radio Observations for Planets, Physics Reports 200, $1-37$.

Drossart, P., Encrenaz, T., Lecacheux, J., Colas, F., \& Lagage, P. 1995. The time sequence of SL9/impacts $\mathrm{H}$ and L from infrared observations. Geophys. Res. Lett. 22, 1769-1772.

Fegley, B. \& Lodders, K. 1994. Chemical models of the deep atmospheres of Jupiter and Saturn. Icarus 110, 117-154.

Field, G. \& Ferrara, A. 1995. The behavior of fragments of Comet Shoemaker-Levy 9 in the atmosphere of Jupiter. Astrophys. J. 438, 957-967.

Field, G., TozzI, G., \& Stanga, R. 1995. Dust as the cause of spots on Jupiter. Astron. Astrophys. 294, L53-L55.

Friedson, A. J., Hoffman, W., Goguen, J., Deutsch, L., Orton, G., Hora, J., Dayal, A., SPitale, J., Wells, W. K., \& Fazio, G. 1995. Thermal infrared lightcurves of the impact of Comet Shoemaker-Levy 9 fragment R. Geophys. Res. Lett. 22, 1569-1572.

Graham, J., De Pater, I., Jernigan, J., Liu, M., \& Brown, M. 1995. W. M. Keck telescope observations of the comet P/Shoemaker-Levy 9 fragment R Jupiter collision. Science 267, $1320-1323$.

Hammel, H., Beebe, R., Ingersoll, A., Orton, G., Mills, J., Simon, A., Chodas, P., Clarke, J., De Jong, E., Dowling, T., Harrington, J., Huber, L., Karkoschka, E., Santori, C., Toigo, A., Yeomans, D., \& West, R. 1995. Hubble Space Telescope 
imaging of Jupiter: atmospheric phenomena created by the impact of comet ShoemakerLevy 9. Science 267, 1288-1296.

IngERSoll, A., AND KANAMORI, H. 1995. Waves from the impacts of Shoemaker-Levy 9 with Jupiter Nature 374, 706-708.

Ingersoll, A., Kanamori, H., \& Dowling, T. 1994. Atmospheric gravity waves from the impact of Shoemaker-Levy 9 with Jupiter Geophys. Res. Lett. 21, 1083-1086.

Kim, S., Ruiz, M., Rieke, G., Rieke, M., MaC Low, M.-M., \& Zahnle, K. 1995. The re-entry shock of the $\mathrm{R}$ fragment of Comet Shoemaker-Levy 9. Nature (submitted).

KNaCke, R. F., Geballe, T. R., Noll, K. S., \& Brooke, T. Y. 1994. Infrared spectra of the R post-impact events of comet Shoemaker-Levy 9. Bull. Amer. Astron. Soc. Special Sessions on SL9 26, 3.25.

Knacke, R. F., Fajardo-Acosta, S. B., Geballe, T. R., \& Noll, K. S. 1995. iaU Colloquium 156 , p. 59.

Lellouch, E., Paubert, G., Moreno, R., Festou, M., Bézard, B., Bockelée-Morvan, D., Colom, P., Crovisier, J., Encrenaz, T., Gautier, D., Marten, A., Despois, D., Strobel, D., \& Sievers, A. 1995. Chemical and thermal response of Jupiter to the impact of comet Shoemaker-Levy 9 . Nature 373, 592-595.

MaC Low, M.-M. \& ZAHNLE, K. 1994. Explosion of Comet Shoemaker-Levy 9 on entry into the Jovian atmosphere, Astrophys. J. Lett. 434, L33-L36.

MCGregor, P., Nicholson, P., \& AlLen, M. 1995. CASPIR observations of the collision of Comet Sheomaker-Levy 9 with Jupiter. Icarus (submitted).

MCKinnon, WM. B., \& Schenk, P. 1995. Estimates of comet fragment masses from impact crater chains on Callisto and Ganymede. Geophys. Res. Lett. 22, 1829-1832.

Meadows, V., Crisp, D., Orton, G., Brooke, T., \& Spencer, J. 1994. AAT observations of Shoemaker-Levy 9 collisions with Jupiter. poster presented at the $26^{\text {th }}$ Ann. Mtg., Div. Planet. Sci., Bethesda MD, Oct. 31-Nov. 4, 1994.

Melosh, H. J., Schneider, N., Zahnle, K., \& Latham, D. 1990. Ignition of global wildfires at the Cretaceous/Tertiary boundary. Nature 343, 251-254.

Moses, J. I., Allen M., \& Gladstone, R. 1995. Post-SL9 sulfur photochemistry on Jupiter, Geophys. Res. Lett. 22, 1597-1600.

Nicholson, P., Gierasch, P., Hayward, T., McGhee, C., Moersch, J., Squyres, S., Van Cleve, J., Matthews, K., Neugebauer, G., Shupe, D., Weinberger, A., Miles, J., \& ConRath, B. 1995a. Palomar observations of the impact of the R fragment of comet P/Shomeaker-Levy 9: Light curves. Geophys. Res. Lett. 22, 1613-1616.

Nicholson, P., Gierasch, P., Hayward, T., McGhee, C., Moersch, J., Squyres, S., Van Cleve, J., Matthews, K., Neugebauer, G., Shupe, D., Weinberger, A., Miles, J., \& Conrath, B. 1995b. Palomar observations of the impact of the R fragment of comet P/Shomeaker-Levy 9: Spectra. Geophys. Res. Lett. 22, 1617-1620.

Noll, K., McGrath, M., Trafton, L., Atreya, S., Caldwell, J., Weaver, H., Yelle, R., BARNET, C., \& EDGINGTON, S. 1995. HST spectroscopic observations of Jupiter after the collision of Comet Shoemaker-Levy 9. Science 267, 1307-1313.

PrinN, R., \& FEgLEY, B. 1987. Bolide Impacts, acid rain, and biospheric traumas at the Cretaceous-Tertiary boundary. Earth Planet. Sci. Lett. 83 1-15.

Shoemaker, E., Hassig, P., \& Roddy, D. 1995. Numerical modelling of Shoemaker-Levy 9 impacts as a framework for interpreting observations. Geophys. Res. Lett. 22, 1825-1828.

Solem, J. C. 1994. Density and size of comet Shoemaker-Levy 9 deduced from a tidal breakup model. Nature 370, 349-351.

Sprague, A., Buoraker, G., Hunten, D., Witteborn, F., Kozlowski, R., \& Wooden, D. 1996. Water brought into Jupiter's atmosphere by fragments $R$ and $W$ of Comet SL-9. Icarus (in press).

STOKER, C. 1986. Moist convection: A mechanism for producing the vertical structure of the Jovian equatorial plumes. Icarus 67, 106-125. 
Takata, T., O'Keefe, J. D., Ahrens, T. J., \& Orton, G. 1994. Comet Shoemaker-Levy 9: Impact on Jupiter and plume evolution. Icarus 109, 3-19.

Takata, T., Ahrens, T. J., \& Harris, A. 1995. Comet Shoemaker-Levy 9: Fragment and progenitor impact energy. Geophys. Res. Lett. 22, 2433-2436.

Watanabe, J., Yamashita, T., Hasegawa, T., Takeuchi, S., Abe, M., Hirota, Y., NishiHARA, E., OKUMURA, S., \& MORI, A. 1995. Near-IR observation of cometary impacts to Jupiter: Brightness variation of the impact plume of fragment K. Publ. Astron. Soc. Jpn. 47, L21-L24.

West, R., Karkoschka, E., Friedson, A., Seymour, M., Baines, K., \& Hammel, H. 1995. Impact debris particles in Jupiter's stratosphere. Science 267, 1296-1301.

Yabe, T., Xiao, F., Zhang, D., Sasaki, S., Abe, Y., Kobayashi, N., \& Terasawa, T. 1994. Effect of EOS on break-up of Shoemaker-Levy 9 entering jovian atmosphere. J. Geomag. Geolectr. 46, 657-662.

Young, R., ZaHnLe, K., \& MAC Low M.-M., 1995. Nonlinear propagating features in the stratosphere of Jupiter generated by the impact of SL-9. Bull. Amer. Astrom. Soc., (in press).

ZAhnLE, K. 1990. Atmospheric chemistry by large impacts. In Global Catastrophes in Earth History (eds. V. Sharpton \& P. Ward). Geol. Soc. Am. Spec. Pap. 247 pp. 271-P288.

ZahnLe, K., \& MAC Low, M.-M. 1994. The collision of Jupiter and Comet Shoemaker-Levy 9. Icarus 108, 1-17.

ZahNLE, K., \& MAC Low, M.-M. 1995. A simple model for the light curve generated by a Shoemaker-Levy 9 impact. J. Geophys. Res. 100, 16885-16894.

Zahnle, K., MaC Low, M.-M., Lodders, K., \& Fegley, B. 1995. Sulfur chemistry in the wake of Shoemaker-Levy 9. Geophys. Res. Lett. 22, 1593-1596.

Zel'Dovich, IA. B., \& RAIZER, YU. P. 1967. Physics of Shock Waves and High Temperature Hydrodynamic Phenomena Academic. 